\title{
THE CONSTRUCTION OF HARMONIC MAPS INTO COMPLEX GRASSMANNIANS
}

\author{
F. E. BURSTALL \& J. C. WOOD \\ To Professor J. Eells on his sixtieth birthday
}

\section{Introduction}

A. Background. In [17], following work of A. M. Din and W. J. Zakrzewski [9] and V. Glaser and R. Stora [20], J. Eells and the second author described, in terms of holomorphic maps, all harmonic maps (or, equivalently, minimal branched immersions) of the Riemann sphere $S^{2}$ to a complex projective space $\mathbf{C} P^{n}$ and all harmonic maps from a two-torus $T^{2}$ to $\mathbf{C} P^{n}$ of nonzero degree. (For the $S^{2}$ case see also D. Burns [3], and for a moving frames interpretation, S.-S. Chern and J. Wolfson [7], [32].) The harmonic maps were obtained by successive differentiations of a holomorphic map; this process gave all harmonic maps from any Riemannn surface to $\mathbf{C} P^{n}$ satisfying a certain "isotropy" property of orthogonality of iterated $(1,0)$ and $(0,1)$ derivatives. The vanishing of a sequence of holomorphic differentials (cf. [34]) then guaranteed isotropy in the $S^{2}$ and $T^{2}$ cases showing that all harmonic maps had been obtained.

Regarding $\mathbf{C} P^{n}$ as the complex Grassmannian $G_{1, n+1}$ of (complex) 1-planes in Euclidean $(n+1)$-space $\mathbf{C}^{n+1}$, it was natural to try to extend these results to give a description of all harmonic maps from $S^{2}$ to a complex Grassmannian in terms of "holomorphic data". In [19] S. Erdem and the second author showed how to construct all harmonic maps from any Riemann surface to a complex Grassmannian $G_{k, n}$ which satisfy a "strong isotropy" property, however, for $k>1$, this did not give all harmonic maps from the Riemann sphere $S^{2}$ to $G_{k, n}$ (for further developments and related work see [10], [18], [21]). In [25] J. Ramanthan succeeded in describing all harmonic maps from the Riemann sphere to $G_{2,4}$ in terms of "holomorphic data" by which we shall henceforth mean holomorphic maps into a Grassmannian and holomorphic sections of fibre bundles over the domain. A. R. Aithal then dealt with the case

Received June 17, 1985. 
$G_{2,5}$ [2] and also gave a description of certain harmonic maps from $S^{2}$ to a quaternionic projective space $\mathbf{H} P^{n}$ [1]. The first author [4] gave a twistor interpretation of Ramanathan's result and a description of all harmonic maps from $S^{2}$ to a complex Grassmannian in terms of maps into a twistor bundle $Z$ over $G_{k, n}$ which are holomorphic with respect to a certain nonintegrable complex structure on $Z$. (For other twistor space constructions of harmonic maps see [5], [14], [15], [26], [29], [30].)

It was to try to understand this description in more concrete terms that the present work was started in June 1984. Then in a lecture [33] J. Wolfson outlined his work with S.-S. Chern on the construction of all harmonic maps of the Riemann sphere into complex Grassmannians $G_{2, n}$ from holomorphic data (his description was confined by the time constraint to $G_{2,6}$ ), an announcement by Chern and Wolfson of the case $G_{2, n}$ ( $n$ arbitrary) appears in [8].

B. Results. We develop a technique of analyzing harmonic maps from a Riemann surface into a complex Grassmannian using "diagrams" which establishes several results, firstly a proof of the result above of Chern and Wolfson describing all harmonic maps from the Riemann sphere to $G_{2, n}$ in terms of holomorphic data (Theorem 3.3). This theorem says that any harmonic map $\phi: S^{2} \rightarrow G_{2, n}$ can be obtained from a harmonic map $\phi_{0}: S^{2} \rightarrow G_{2, n}$ of simple type describable in terms of holomorphic maps (in fact, with $\phi_{0}$ or $\phi_{0}^{\perp}$ strongly isotropic) by a finite number of "replacements". A replacement consists of replacing a holomorphic part of the map by its image under a differentiation; the choice of such a holomorphic part is equivalent to the choice of a holomorphic section of a $\mathrm{C} P^{1}$ bundle over $S^{2}$. Thus, as in Chern-Wolfson [8], any harmonic map is determined by a collection of holomorphic maps and holomorphic sections of $\mathbf{C} P^{1}$ bundles. Secondly we give a (rather more complicated) description in the same spirit of all harmonic maps from the Riemann sphere into $G_{k, n}(k=3,4,5)$ (Theorem 4.2). Thirdly, we give some results involving degree including a simple description of all harmonic maps of degree 0 or \pm 1 from $S^{2}$ to $G_{2,4}$ (Theorem 5.11) and of all "inclusive" [14], [29] harmonic maps from $S^{2}$ to $G_{2, n}$ and $T^{2}$ to $G_{2, n}$ of odd degree leading to a slightly more explicit version (Theorem 5.8) of a result of Aithal [1] on harmonic maps into quaternionic projective space; also some extensions of the results of Ramanathan [25] and Aithal to Riemann surfaces other than $S^{2}$ (Theorems 5.10 and 5.15, and Corollary 5.16).

C. Methods. The main technique is to use a "diagram" which is a directed graph showing the relationships under differentiations of various subbundles representing the original map of a Riemann surface into a Grassmannian and related maps (see $\$ 1$ ). The use of diagrams was suggested to us by $\mathbf{S}$. Salamon (cf. [29], [30]); we provide some vital new ingredients including a criterion for 
holomorphicity of the edges of the graph (Proposition 1.5) which enables us to construct holomorphic differentials very simply by finding suitable circuits. The vanishing of any holomorphic differential on the Riemann sphere then allows us to "improve" an arbitrary harmonic map by successively replacing a suitable holomorphic part of it by its image under a differentiation until we reach a harmonic map of simple type describable in terms of holomorphic data. The procedure can then be reversed to obtain an arbitrary harmonic map from one of this simple type by successive replacements leading to Theorems 3.3 and 4.2. The results of $\$ 5$ also employ the diagram technique but now use degree conditions to establish vanishing of holomorphic differentials. It is hoped that our methods will prove useful in the further study of harmonic maps into a complex Grassmannian.

D. Acknowledgments. We should like to thank J. Ramanathan, A. R. Aithal and J. Wolfson for informing us of their work, J. Eells and J. Rawnsley for comments on this work, and, especially, S. Salamon for suggesting that his diagrams could be useful in this work and pointing out a correction to Proposition 1.6.

Lastly, we mention some recent work of $\mathrm{K}$. Uhlenbeck [31] discussing harmonic maps into complex Grassmannians and the unitary group. Here harmonic maps into the unitary group are described in terms of holomorphic data in a way related to this paper.

\section{Preliminaries}

A. Let $\phi:(M, g) \rightarrow(N, h)$ be a smooth map of Riemannian manifolds. The energy of $\phi$ over a compact domain $D$ of $M$ is defined by

$$
E(\phi ; D)=\frac{1}{2} \int_{D} \text { Trace } \phi^{*} h d v_{g}
$$

where $d v_{g}$ is the volume element on $(M, g)$.

A map $\phi:(M, g) \rightarrow(N, h)$ is harmonic if, for each compact $D \subset M, \phi$ extremizes the energy $E(\phi ; D)$ with respect to all variations supported in $D$. Then $\phi$ is harmonic iff it satisfies the associated Euler-Lagrange equations

$$
\tau_{\phi}=\operatorname{Trace} \nabla d \phi=0
$$

where $\nabla$ denotes the connection on $T^{*} M \otimes \phi^{-1} T N$ induced by the Levi-Civita connections on $M$ and $N$ respectively. The (nonlinear) differential operator $\tau$ is called the tension field. (For information on harmonic maps see [12], [13].)

Now suppose that $M$ is a Riemann surface with isothermal coordinate $(U, z)$. In this case the tension field of $\phi: M \rightarrow N$ is given on $U$, up to a 
ccnformal factor, by

$$
\left(\phi^{-1} \nabla_{\partial / \partial \bar{z}}^{N}\right) d \phi\left(\frac{\partial}{\partial z}\right)=\left(\phi^{-1} \nabla_{\partial / \partial z}^{N}\right) d \phi\left(\frac{\partial}{\partial \bar{z}}\right) .
$$

Further, if $(N, h)$ is an almost complex manifold, the type decomposition of $T^{\mathrm{C}} N$ induces a decomposition of the differential of $\phi$ :

$$
d \phi=\partial^{(1,0)} \phi+\partial^{(0,1)} \phi
$$

where $\partial^{(1,0)} \phi \in C^{\infty}\left(T_{C}^{*} M \otimes \phi^{-1} T^{(1,0)} N\right)$ and $\partial^{(0,1)} \phi \in C^{\infty}\left(T_{C}^{*} M \otimes \phi^{-1} T^{(0,1)} N\right)$.

Proposition 0.1. Let $\phi:(M, g) \rightarrow(N, h)$ be a smooth map of a Riemann surface into a Kähler manifold. Then $\phi$ is harmonic if and only if

$$
\left(\phi^{-1} \nabla_{\partial / \partial \bar{z}}^{N}\right) \partial^{(1,0)} \phi(\partial / \partial z) \equiv 0
$$

if and only if

$$
\left(\phi^{-1} \nabla_{\partial / \partial z}^{N}\right) \partial^{(1,0)} \phi(\partial / \partial \bar{z}) \equiv 0 .
$$

Proof. If $\phi$ is harmonic, (4) and (4a) follow immediately from (2) since the Kähler condition implies that $\phi^{-1} \nabla^{N}$ preserves the type decomposition of $T^{\mathbf{C}} N$.

For the converse: (4) or (4a) implies that $\tau_{\phi}^{(1,0)}=0$ whence $\tau_{\phi}$ vanishes since it is real.

Remark. The Kähler condition on $N$ can be considerably relaxed (see [24]).

B. Let $G_{k, n}$ denote the Grassmannian of complex $k$-planes in $\mathbf{C}^{n} . G_{k, n}$ is a Kähler manifold (in fact it is a Hermitian symmetric space). Let $T \rightarrow G_{k, n}$ denote the tautological subbundle of $G_{k, n} \times \mathbf{C}^{n}$ whose fibre at $W \in G_{k, n}$ is $W$ itself.

As is well known, there is a natural isomorphism $h: T^{(1,0)} G_{k, n} \rightarrow L\left(T, T^{\perp}\right)$ given by

$$
h(Z) \sigma=\pi_{T^{\perp}}(Z \cdot \sigma)
$$

for $\sigma$ a local section of $T$ and $Z \in T^{(1,0)} G_{k, n}$. Here $\pi_{T^{\perp}}$ denotes orthogonal projection onto $T^{\perp}$. If $T^{(1,0)} G_{k, n}$ is equipped with the Levi-Civita connection and $L\left(T, T^{\perp}\right)$ with the connection induced by the flat connection on $G_{k, n} \times \mathbf{C}^{n}$, then $h$ is connection-preserving.

For more details on the geometry of $G_{k, n}$, see the following section.

\section{Subbundles of $M^{2} \times C^{n}$ and diagrams}

Let $M^{2}$ be a Riemann surface.

A. We identify a smooth map $\phi: M^{2} \rightarrow G_{k, n}$ with a subbundle $\phi$ of the trivial bundle $\underline{\mathbf{C}}^{n}=M^{2} \times \mathbf{C}^{n}$ of rank $k$ which has fibre at $x \in M$ given by 
$\underline{\phi}_{x}=\phi(x)$. Thus $\underline{\phi}=\phi^{-1} T$. Conversely any rank $k$ subbundle of $\underline{\mathbf{C}}^{n}$ induces a $\operatorname{map} M^{2} \rightarrow G_{k, n}$.

Observe that $\phi$ is a holomorphic (resp. antiholomorphic) subbundle of $\underline{\mathbf{C}}^{n}$ if and only if $\phi: \bar{M}^{2} \rightarrow G_{k, n}$ is a holomorphic (resp. antiholomorphic) map.

Definition. A rank $k$ subbundle $\underline{\phi}$ of $\underline{\mathbf{C}}^{n}$ is said to be harmonic if $\phi$ : $M^{2} \rightarrow G_{k, n}$ is harmonic.

Any subbundle $\phi$ of $\underline{\mathbf{C}}^{n}$ inherits a metric, denoted $\langle,\rangle_{\phi}$, and connection, denoted $\nabla_{\phi}$, from the flat metric $\langle$,$\rangle and connection, \partial$, on $\underline{\mathbf{C}}^{n}$. Explicitly,

$$
\begin{array}{ll}
\langle v, w\rangle_{\phi}=\langle v, w\rangle, & v, w \in \underline{\phi}_{x}, x \in M ; \\
\left(\nabla_{\phi}\right)_{Z} v=\pi_{\phi} \partial_{Z} v, \quad v \in C^{\infty}(\underline{\phi}), Z \in T M .
\end{array}
$$

Here $\pi_{\phi}: \underline{\mathbf{C}}^{n} \rightarrow \phi$ denotes orthogonal projection.

Now let $(U, z)$ be an isothermal coordinate on $M$. We denote $\left(\nabla_{\phi}\right)_{\partial / \partial z}$ and $\left(\nabla_{\phi}\right)_{\partial / \partial z}$ by $\nabla_{\phi}^{\prime}$ and $\nabla_{\phi}^{\prime \prime}$, respectively. Similarly, for the connection on $\underline{\mathbf{C}}^{n}$ we denote $\partial_{\partial / \partial z}$, and $\partial_{\partial / \partial \bar{z}}$ by $\partial^{\prime}$ and $\partial^{\prime \prime}$, respectively. $\partial^{\prime \prime}$.

Note. $\underline{\phi}$ is a holomorphic subbundle if and only if $C^{\infty}(\underline{\phi})$ is closed under

Important Remark. We shall usually work in an isothermal chart but it should be noted that all our constructions will be independent of the choice of such a chart. Indeed, we could work without such a choice but only with a loss of clarity due to the increased complication of notation.

B. We recall the fundamental theorem of Koszul-Malgrange [23]:

Theorem 1.1. Let $E \rightarrow M$ be a complex vector bundle with connection $\nabla$ over a Riemann surface $M$. Then there is a unique complex structure on $E$ for which $E \rightarrow M$ is a holomorphic vector bundle and a local $C^{\infty}$ section $\sigma$ is holomorphic if and only if

$$
\nabla_{Z} \sigma=0 \quad \text { for all } Z \in T^{(0,1)} M .
$$

Remark. If $\underline{\phi}$ is a holomorphic subbundle of $\underline{\mathbf{C}}^{n}$, then $\partial^{\prime \prime}=\nabla_{\phi}^{\prime \prime}$ so that the Koszul-Malgrange complex structure on $\underline{\phi}$ induced by $\nabla_{\phi}$ coincides with the complex structure on $\underline{\phi}$ qua holomorphic subbundle of $\underline{\mathbf{C}}^{n}$.

Henceforth we equip all subbundles $\phi$ of $\underline{\mathbf{C}}^{n}$ with the complex structure induced by $\nabla_{\phi}$ via Theorem 1.1. By the above remark this choice of complex structure is unambiguous.

Now let $A: \underline{\phi} \rightarrow \underline{\psi}$ be a vector bundle morphism. Observe that $A$ is a holomorphic section of $L(\underline{\phi}, \underline{\psi})$ if and only if $A \circ \nabla_{\phi}^{\prime \prime}=\nabla_{\psi}^{\prime \prime} \circ A$ for all choices of isothermal coordinate on $\bar{M}$.

We now collect together some useful properties of sections of $L(\underline{\phi}, \underline{\psi})$, the proofs of which are left as an (easy!) exercise for the reader. 
Proposition 1.2. Let $A: \underline{\phi} \rightarrow \underline{\psi}$ be a section of $L(\underline{\phi}, \underline{\psi})$.

(i) If $A$ is holomorphic and $\underline{\alpha} \subset \underline{\phi}$ is a holomorphic subbundle, then $A \mid \underline{\alpha}$ : $\underline{\alpha} \rightarrow \Psi$ is holomorphic.

(ii) Let $\underline{\beta} \subset \underline{\psi}$ be an antiholomorphic subbundle of $\underline{\psi}$ with $\pi: \underline{\psi} \rightarrow \underline{\beta}$ denoting orthogonal projection on $\underline{\beta}$. If $A$ is holomorphic, then $\pi \circ A: \underline{\phi} \rightarrow \underline{\beta}$ is holomorphic.

(iii) Let $\underline{\gamma} \subset \underline{\phi}$ be a subbundle with $\underline{\gamma}_{x}{ }^{\perp} \subset \operatorname{ker} A_{x}$ for all $x \in M$. Then $A$ is holomorphic if and only if $A \mid \underline{\gamma}: \underline{\gamma} \rightarrow \Psi$ is holomorphic.

(iv) Let $\underline{\delta} \subset \underline{\psi}$ be a subbundle of $\underline{\psi}$ containing the image of each $A_{x}, x \in M$. If $A$ is holomorphic, then $A: \underline{\phi} \rightarrow \underline{\delta}$ is holomorphic. Conversely, if $\underline{\delta}$ is a holomorphic subbundle of $\underline{\psi}$ and $A: \underline{\phi} \rightarrow \underline{\delta}$ is holomorphic, then $A$ is holomorphic.

C. Let $\underline{\phi}, \underline{\psi}$ be mutually orthogonal subbundles of $\underline{\mathbf{C}}^{n}$. We define the $\partial^{\prime}$-second fundamental form of $\underline{\phi}$ in $\phi \oplus \underline{\psi}$ as the (locally defined) vector bundle morphism $A_{\phi, \psi}^{\prime}: \underline{\phi} \rightarrow \underline{\psi}$ given by

$$
A_{\phi, \psi}^{\prime}(v)=\nabla_{\psi+\phi}^{\prime} v-\nabla_{\phi}^{\prime} v, \quad v \in C^{\infty}(\underline{\phi}) \text {. }
$$

Thus, if, for subbundles $\underline{\alpha} \subset \underline{\beta} \subset \underline{\mathbf{C}}^{n}, \pi_{\beta, \alpha}$ denotes the orthogonal projection from $\underline{\beta}$ to $\underline{\alpha}$, we have

$$
A_{\phi, \psi}^{\prime}(v)=\pi_{\phi+\psi, \psi} \circ \nabla_{\phi+\psi}^{\prime} v=\pi_{\mathbf{C}^{n}, \psi} \partial^{\prime} v, \quad v \in C^{\infty}(\underline{\phi})
$$

Similarly, we define the $\partial^{\prime \prime}$-second fundamental form of $\underline{\phi}$ in $\underline{\phi} \oplus \underline{\psi}, A_{\phi, \psi}^{\prime \prime}$ : $\underline{\phi} \rightarrow \underline{\psi}$, by

$$
A_{\phi, \psi}^{\prime \prime}(v)=\nabla_{\psi+\phi}^{\prime \prime} v-\nabla_{\phi}^{\prime \prime} v=\pi_{\mathbf{C}^{n}, \psi} \partial^{\prime \prime} v, \quad v \in C^{\infty}(\underline{\phi})
$$

Observe that $A_{\phi, \psi}^{\prime}$ is the negative of the adjoint of $A_{\psi, \phi}^{\prime \prime}$ viz:

$$
-\left\langle A_{\phi, \psi}^{\prime} v, w\right\rangle_{\phi}=\left\langle v, A_{\psi, \phi}^{\prime \prime} w\right\rangle_{\phi} \text { for } v \in \underline{\phi}_{x}, w \in \underline{\Psi}_{x}, x \in M .
$$

Remark. Of course, $A_{\phi, \psi}^{\prime}$ and $A_{\phi, \psi}^{\prime \prime}$ are strictly only vector bundle morphisms $\underline{\phi}|U \rightarrow \underline{\psi}| U$ for some isothermal chart $(U, z)$. Globally, they give rise to $L(\underline{\phi}, \underline{\Psi})$-valued 1-forms, however, in keeping with our policy of working in an isothermal chart we will usually view these second fundamental forms as vector bundle morphisms.

Of particular importance are the second fundamental forms of $\phi$ in $\mathbf{C}^{n}$ :

$$
A_{\phi}^{\prime}=A_{\phi, \phi^{\perp}}^{\prime}: \underline{\phi} \rightarrow \underline{\phi}^{\perp}, \quad A_{\phi}^{\prime \prime}=A_{\phi, \phi^{\perp}}^{\prime \prime}: \underline{\phi} \rightarrow \underline{\phi}^{\perp},
$$

which, via the isomorphism of $T^{(1,0)} G_{k, n}$ and $L\left(T, T^{\perp}\right)$ of $\S 0$.(B), represent the partial derivatives $\partial^{(1,0)} \phi(\partial / \partial z)$ and $\partial^{(1,0)} \phi(\partial / \partial \bar{z})$, respectively, of $\phi: M^{2} \rightarrow$ $G_{k, n}$. (See the proof of the following lemma.)

Lemma 1.3. Let $\phi: M^{2} \rightarrow G_{k, n}$ be a smooth map.

(a) $\phi$ is holomorphic (respectively antiholomorphic) if and only if

$$
A_{\phi}^{\prime \prime}=0 \quad\left(\text { respectively } A_{\phi}^{\prime}=0\right) \text {. }
$$


By the above observation, the first condition is equivalent to the vanishing of $A_{\phi^{\perp}}^{\prime}$.

(b) $\phi$ is harmonic if and only if $A_{\phi}^{\prime}: \underline{\phi} \rightarrow \underline{\phi}^{\perp}$ is holomorphic, i.e.,

$$
A_{\phi}^{\prime} \circ \nabla_{\phi}^{\prime \prime}=\nabla_{\phi^{\perp}}^{\prime \prime} \circ A_{\phi}^{\prime},
$$

or equivalently, $A_{\phi}^{\prime \prime}: \underline{\phi} \rightarrow \underline{\phi}^{\perp}$ is antiholomorphic.

Proof. (a) $A_{\phi}^{\prime \prime}=0$ if and only if $\partial^{\prime \prime} C^{\infty}(\underline{\phi}) \subset C^{\infty}(\underline{\phi})$ or equivalently, $\underline{\phi}$ is a holomorphic subbundle of $\underline{\mathbf{C}}^{n}$.

(b) Recall the isomorphism $h: T^{(1,0)} G_{k, n} \rightarrow L\left(T, T^{\perp}\right)$ in $\S 0$.(B). $h$ pulls back to a connection preserving isomorphism

$$
\phi^{-1} h: \phi^{-1} T^{(1,0)} G_{k, n} \rightarrow L\left(\underline{\phi}, \underline{\phi}^{\perp}\right)
$$

and it is clear from formula (5) and the above development that

$$
\phi^{-1} h\left(\partial \phi^{(1,0)}(\partial / \partial z)\right)=A_{\phi}^{\prime}, \quad \phi^{-1} h\left(\partial \phi^{(1,0)}(\partial / \partial \bar{z})\right)=A_{\phi}^{\prime \prime} .
$$

Then the first part of (b) follows from Proposition 0.1 since the holomorphicity of $A_{\phi}^{\prime}$ is equivalent to the vanishing of $\nabla^{\prime \prime}\left(A_{\phi}^{\prime}\right)$, where $\nabla$ is the connection on $L\left(\underline{\phi}, \underline{\phi}^{\perp}\right)$. The second part follows similarly.

Remark. Since $-A_{\phi^{\perp}}^{\prime \prime}$ is the adjoint of $A_{\phi}^{\prime}$ and therefore antiholomorphic if $A_{\phi}^{\prime}$ is holomorphic, we see that $\phi$ is harmonic if and only if $\phi^{\perp}$ is harmonic. Of course, this fact is true for any domain since the map $W \mapsto W^{\perp}$ is an isometry $G_{k, n} \rightarrow G_{n-k, n}$.

D. Now let $\underline{\phi}_{1}, \cdots, \underline{\phi}_{s}$ be a set of mutually orthogonal subbundles of $\underline{\mathbf{C}}^{n}$ whose sum is $\underline{\mathbf{C}}^{n}$. We indicate the situation thus: $\underline{\mathbf{C}}^{n}=\underline{\phi}_{1}(\perp) \cdots$ (1) $\underline{\phi}_{s}$. $A_{i, j}^{\prime}$.

Denote the $\partial^{\prime}$-second fundamental form of $\underline{\phi}_{i}$ in $\underline{\phi}_{i} \bar{\oplus}_{\underline{\phi}_{j}}, A_{\phi_{i}, \phi_{j}}^{\prime}: \underline{\phi}_{i} \rightarrow \underline{\phi}_{j}$, by

We call the collection of subbundles $\underline{\phi}_{i}, 1 \leqslant i \leqslant s$, and second fundamental forms $A_{i, j}^{\prime}, 1 \leqslant i \neq j \leqslant s$, a diagram $\left\{\underline{\phi}_{i}, A_{i, j}^{\prime}\right\}$.

We represent such a diagram by a directed graph with vertices $\underline{\phi}_{1}, \cdots, \underline{\phi}_{s}$ and for each ordered pair $(i, j)$ an edge from $\underline{\phi}_{i}$ to $\underline{\phi}_{j}$ representing $A_{i, j}^{\prime}$. The absence of a given edge in the graph will indicate the vanishing of the corresponding second fundamental form.

Remarks. (i) The use of directed graphs in this context was suggested to us by S. Salamon (cf. [29], [30]).

(ii) The $s$-tuple $\left(\underline{\phi}_{1}, \cdots, \underline{\phi}_{s}\right)$ may be thought of as a map into a flag manifold, $\Phi: M^{2} \rightarrow F$. If there is at most one edge joining each pair of vertices in the corresponding graph, then $\Phi$ is holomorphic with respect to some almost complex structure on $F$. For the relationship between almost complex structures on flag manifolds and harmonic maps into $G_{k, n}$ see [4]. 
Convention. Henceforth we shall use the term diagram to mean either the collection $\left\{\underline{\phi}_{i}, A_{i, j}^{\prime}\right\}$ or a directed graph representing it.

We can use the diagram to determine the properties of various subbundles. For example:

Proposition 1.4. Given a diagram $\left\{\underline{\phi}_{i}, A_{i, j}^{\prime}\right\}$,

(a) $\underline{\phi}_{i}$ is holomorphic if and only if there are no edges entering $\underline{\phi}_{i}$, i.e., $A_{j, i}^{\prime}=0$ for all $i \neq j$.

(b) $\underline{\phi}_{i}$ is antiholomorphic if and only if there are no edges leaving $\underline{\phi}_{i}$, i.e., $A_{i, j}^{\prime}=\overline{0}$ for all $i \neq j$.

Proof. (b) $A_{\phi_{i}}^{\prime}=\sum_{i \neq j} A_{i, j}^{\prime}$ whence the proposition is immediate from Proposition 1.3(a)

(a) $A_{j, i}^{\prime}=0$ if and only if $A_{i, j}^{\prime \prime}=0$ and $A_{\phi_{i}}^{\prime \prime}=\sum_{i \neq j} A_{i, j}^{\prime \prime}$ whence the result follows from Proposition 1.3(a). q.e.d.

It will be of great importance in the sequel to be able to detect whether a given $\partial^{\prime}$-second fundamental form $A_{i, j}^{\prime}: \underline{\phi}_{i} \rightarrow \underline{\phi}_{j}$ is holomorphic. For this, a useful criterion is given by

Proposition 1.5. Given a diagram $\left\{\phi_{i}, A_{i, j}^{\prime}\right\}, A_{i, j}^{\prime}: \underline{\phi}_{i} \rightarrow \underline{\phi}_{j}$ is holomorphic if the diagram contains no configurations of the following forms:

(i)

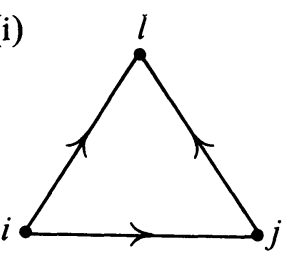

(ii)

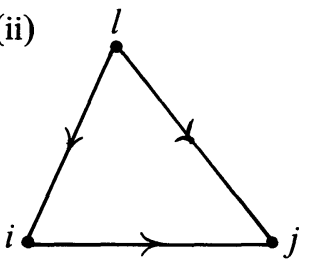

(iii)

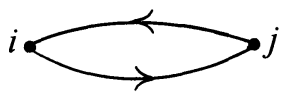

Proof. Let $\pi_{l}: \underline{\mathbf{C}}^{n} \rightarrow \underline{\phi}_{l}$ denote projection onto $\underline{\phi}_{l}, 1 \leqslant l \leqslant k$. We have $\partial^{\prime \prime} C^{\infty}\left(\underline{\phi}_{i}\right) \subset C^{\infty}\left(\underline{\phi}_{i}+\sum_{s=1}^{t} \underline{\phi}_{i_{s}}\right)$, where $j \notin\left\{i_{1}, \cdots, i_{t}\right\}$ since there is no configuration of type (iii).

Further $\partial^{\prime} C^{\infty}\left(\underline{\phi}_{i_{s}}\right) \subset C^{\infty}\left(\underline{\phi}_{j}^{\perp}\right)$ since there are no configurations of type (ii). Thus

$$
\pi_{j} \partial^{\prime} \partial^{\prime \prime}=\pi_{j} \partial^{\prime} \pi_{i} \partial^{\prime \prime}=A_{i, j}^{\prime} \circ \nabla_{\phi_{i}}^{\prime \prime}
$$

Similarly

$$
\nabla_{\phi_{j}}^{\prime \prime} \circ A_{i, j}^{\prime}=\pi_{j} \partial^{\prime \prime} \partial^{\prime}
$$

whence

$$
\nabla_{\phi_{j}}^{\prime \prime} \circ A_{i, j}^{\prime}=A_{i, j}^{\prime} \circ \nabla_{\phi_{i}}^{\prime \prime},
$$

and $A_{i, j}^{\prime}$ is holomorphic. q.e.d. 
As an application of Proposition 1.5 we give a sufficient condition for a vertex to be harmonic. (We thank S. Salamon for correcting our original version of this.)

Proposition 1.6. Given a diagram $\left\{\underline{\phi}_{i}, A_{i, j}^{\prime}\right\}, \underline{\phi}_{i}$ is harmonic if there are no configurations in the diagram of the form:
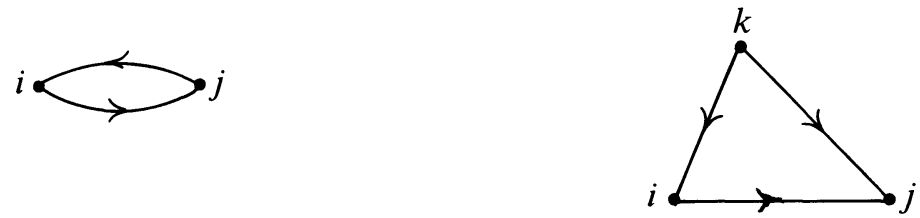

Proof. Put $\underline{\alpha}=\sum\left\{\underline{\phi}_{j}: A_{i, j}^{\prime} \not \equiv 0\right\}$ and $\underline{\beta}=\underline{\alpha}^{\perp} \cap \underline{\phi}_{i}^{\perp}$. Then $A_{\phi_{i}, \beta}^{\prime}=0$ by definition of $\underline{\alpha}$ and $\underline{\beta}, A_{\alpha, \phi_{i}}^{\prime}=0$ since there are no configurations of type (i) and $A_{\beta, \alpha}^{\prime}=0$ since there are no configurations of type (ii). Thus we have a diagram

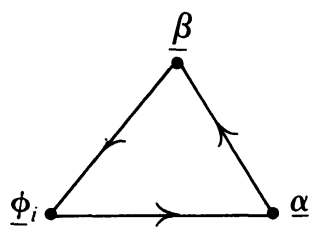

from which we see that $\underline{\alpha}$ is a holomorphic subbundle of $\underline{\phi}_{i}^{\perp}$ and, from Proposition 1.5, $A_{\phi_{i}, \alpha}^{\prime}$ is holomorphic. It then follows from Proposition 1.2 that $A_{\phi_{i}}^{\prime}$ is holomorphic, since $\operatorname{Im} A_{\phi_{i}}^{\prime} \subset \underline{\alpha}$ and thus $\phi_{i}$ is harmonic.

Examples. (i) A $\partial^{\prime}$-pair $(V, X)$ in the sense of Erdem-Wood [19] is a pair of holomorphic subbundles of $\underline{\mathrm{C}}^{n}$ such that (i) $V \subset X$ and (ii) $\partial^{\prime} C^{\infty}(V) \subset C^{\infty}(X)$. It is easy to see that the decomposition $\underline{\mathrm{C}}^{n}=V$ (1) $V^{\perp} \cap X(1) X^{\perp}$ has diagram

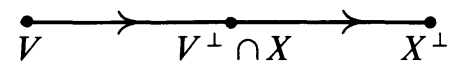

and from Proposition 1.6 we see that $V^{\perp} \cap X$ is harmonic. Thus we recover the result of Erdem-Wood that for any $\partial^{\prime}$ pair $(V, X), V^{\perp} \cap X$ defines a harmonic map.

Conversely, given a diagram of the form

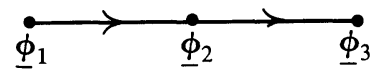

it is clear from Proposition 1.4 and the defintions that $\left(\phi_{1}, \phi_{3}^{\perp}\right)$ is a $\partial^{\prime}$-pair. 
(ii) Consider the diagram

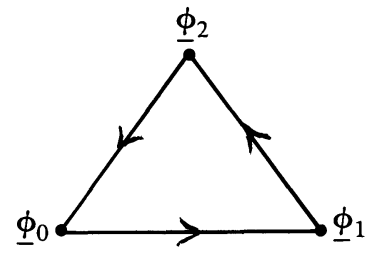

It is immediate from Proposition 1.6 that all $\underline{\phi}_{i}$ are harmonic. Such diagrams correspond to maps into the flag manifold

$$
F=\frac{U(n)}{U\left(r_{1}\right) \times U\left(r_{2}\right) \times U\left(n-r_{1}-r_{2}\right)},
$$

$\Phi=\left(\phi_{0}, \phi_{1}, \phi_{2}\right): M^{2} \rightarrow F$ which are holomorphic with respect to the unique (up to orientation) nonintegrable almost complex structure on $F$. In this context, the vanishing of one of the three edges corresponds to the horizontality of $\Phi$ with respect to one of the homogeneous fibrations of $F$ onto $G_{r_{1}, n}$, $G_{r_{2}, n}$ or $G_{n-r_{1}-r_{2}, n}$.

For extensions of this result and an interpretation in the twistor context see Burstall and Eells-Salamon [4], [15].

E. Recall that the second fundamental forms $A_{\phi, \psi}^{\prime}: \phi \rightarrow \psi$ were defined with respect to an isothermal chart. However, like all constructions in this paper, there is a corresponding global construction. In fact, consider the globally defined section $\mathscr{A}_{\phi, \psi}^{\prime}$ of $T_{(1,0)}^{*} M \otimes L(\underline{\phi}, \underline{\Psi})$ given by

$$
\mathscr{A}_{\phi, \psi}^{\prime}(Z) v=\left(\nabla_{\phi+\psi}\right)_{Z} v-\left(\nabla_{\phi}\right)_{Z} v, \quad v \in C^{\infty}(\underline{\phi}), Z \in T^{(1,0)} M .
$$

Clearly $\mathscr{A}_{\phi, \psi}^{\prime}=d z \otimes A_{\phi, \psi}^{\prime}$ and so $\mathscr{A}_{\phi, \psi}^{\prime}$ is holomorphic if and only if each $A_{\phi, \psi}^{\prime}$ is.

The following lemma follows immediately from Leibniz' rule:

Lemma 1.7. Let $\mathscr{A}, \mathscr{B}$ be holomorphic sections of $\otimes{ }^{k} T_{(1,0)}^{*} M \otimes L(E, F)$, $\otimes^{\prime} T_{(1,0)}^{*} M \otimes L(F, G)$, respectively, where $E, F, G$ are holomorphic vector bundles. Then composition defines a holomorphic section $\mathscr{B} \circ \mathscr{A}$ of $\otimes^{k+l} T_{(1,0)}^{*} M$ $\otimes L(E, G)$.

The following proposition, known to several authors, first appeared in Ramanathan [25].

Proposition 1.8. Let $S^{2}$ denote the Riemann sphere and let $\mathscr{A}$ denote a holomorphic section of $\otimes{ }^{k} T_{(1,0)}^{*} S^{2} \otimes L(E, E)$, where $E \rightarrow S^{2}$ is a holomorphic vector bundle. Then writing $\mathscr{A}$ as $d z^{k} \otimes A, A$ is nilpotent on each fibre and in particular cannot be an isomorphism on any fibre. 
Proof. The coefficients of the characteristic polynomial of $A$ give rise to globally defined holomorphic differentials on $S^{2}$. Since $S^{2}$ admits no nonzero holomorphic differentials it follows that $A$ is nilpotent. q.e.d.

Applying Propositions 1.7 and 1.8 to $\partial^{\prime}$-second fundamental forms we have

Proposition 1.9. Let $\left\{\underline{\phi}_{i}, A_{i, j}\right\}$ be a diagram. Suppose there exist $\left\{i_{1}, \cdots, i_{k}\right\}$ such that $A_{i_{1}, i_{2}}^{\prime}, \cdots, A_{i_{k}, i_{1}}^{\prime}$ are holomorphic. (We say $\underline{\phi}_{i_{1}}, \cdots, \underline{\phi}_{i_{k}}, \underline{\phi}_{i_{1}}$ form a holomorphic circuit.) Then their composition gives rise to a holomorphic section of $\otimes{ }^{k} T_{(1,0)}^{*} M \otimes L\left(\underline{\phi}_{i_{1}}, \underline{\phi}_{i_{1}}\right)$.

Further if $M=S^{2}$, then $\bar{A}_{i_{k}, i_{1}}^{\prime}{ }^{\circ} \ldots \circ A_{i_{1}, i_{2}}^{\prime}$ is nilpotent and in particular is not an isomorphism on any fibre.

Corollary 1.10. If $M=S^{2}$ and $\operatorname{dim} \underline{\phi}_{i_{j}}=1$ for all $j$, then at least one $A_{i_{j}, i_{j+1}}^{\prime}$ must vanish.

Example (cf. [25]). Let $\phi: S^{2} \rightarrow G_{r, n}$ be harmonic. Then the map $A_{\phi^{\perp}}^{\prime} \circ A_{\phi}^{\prime}$ : $\phi \rightarrow \phi$ is nilpotent.

Example. Let $M=S^{2}$ and consider the diagram:

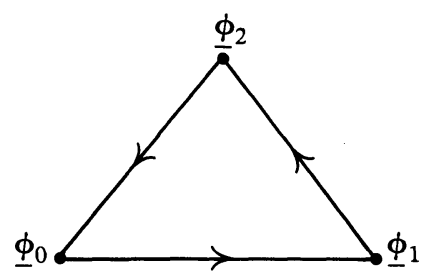

From Proposition 1.5 we see that $\underline{\phi}_{0}, \underline{\phi}_{1}, \underline{\phi}_{2}, \underline{\phi}_{0}$ is a holomorphic circuit, so if each $\underline{\phi}_{i}$ has rank one we conclude from the corollary that the diagram reduces to

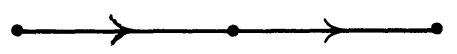

and so one of the $\underline{\phi}_{i}$ is holomorphic and one antiholomorphic. We will extend this example at the end of the following section.

\section{New harmonic maps from old}

In this section we exhibit a technique, 'replacement', for producing new harmonic maps from a given harmonic map.

A.

Lemma 2.1. Let $\mathbf{C}^{n}=\underline{\alpha}(1) \underline{\beta}(1) \underline{\gamma}$ and suppose that $A_{\alpha, \beta}^{\prime}: \underline{\alpha} \rightarrow \underline{\beta}$ and $A_{\beta, \gamma}^{\prime}$ : $\underline{\beta} \rightarrow \underline{\gamma}$ both vanish. Then $\underline{\alpha}$ is harmonic if and only if $\underline{\gamma}$ is harmonic. 
Remark. The diagram for this situation is:

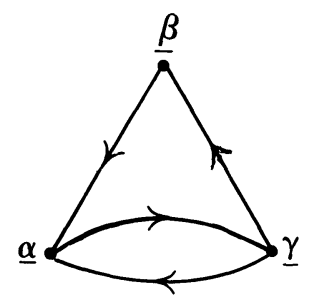

Proof. From Lemma 1.3(b) $\underline{\alpha}$ is harmonic if and only if $A_{\alpha}^{\prime}$ is holomorphic. Since $\underline{\gamma}$ is a holomorphic subbundle of $\underline{\alpha}^{\perp}$ and $\operatorname{Im} A_{\alpha}^{\prime} \subset \underline{\gamma}$, from Proposition 1.2(iv) we have $A_{\alpha}^{\prime}$ is holomorphic if and only if $A_{\alpha, \gamma}^{\prime}$ is holomorphic.

Since $\underline{\beta} \subset \operatorname{ker} A_{\alpha+\beta}^{\prime}$ we have from Proposition 1.2(iii) that $A_{\alpha+\beta}^{\prime}=A_{\alpha+\beta, \gamma}^{\prime}$ is holomorphic if and only if $A_{\alpha+\beta, \gamma}^{\prime} \mid \underline{\alpha}=A_{\alpha, \gamma}^{\prime}$ is holomorphic. Thus $\underline{\alpha}$ is harmonic if and only if $\underline{\alpha}+\underline{\beta}$ is harmonic if and only if $(\underline{\alpha}+\underline{\beta})^{\perp}=\underline{\gamma}$ is harmonic. q.e.d.

To construct new harmonic maps from old, we need the following

Proposition 2.2 ( $c f . W u$ [35]). "Filling out zeros." Let $E, F$ be holomorphic vector bundles over $M^{2}$ and $\mathscr{A}=A \otimes d z^{k}$ a holomorphic section of $\otimes{ }^{k} T_{(1,0)}^{*} M$ $\otimes L(E, F)$. Let $t=\max _{x \in M}\left(\operatorname{dim} \operatorname{Im} A_{x}\right)$. Then there are unique holomorphic subbundles $\underline{\alpha}$ and $\underline{\beta}$ of $E$ and $F$ respectively with $\operatorname{rank} \underline{\alpha}=\operatorname{rank} E-t, \operatorname{rank} \underline{\beta}=t$ such that

$$
\left.\begin{array}{l}
\underline{\alpha}_{x} \supseteq \operatorname{ker} A_{x} \\
\underline{\beta}_{x} \subseteq \operatorname{Im} A_{x}
\end{array}\right\} \text { with equality for all } x \text { such that } \operatorname{dim} \operatorname{Im} A_{x}=t .
$$

Remark. As the proof will show, the set $\left\{x: \operatorname{dim} \operatorname{Im} A_{x}<t\right\}$ is isolated.

Proof. Observe that $\wedge^{t} A$ is a holomorphic local section of $L\left(\bigwedge^{t} E, \Lambda^{t} F\right)$ and thus has isolated zeros or vanishes identically. Thus by a simple connectedness argument we have that $\operatorname{dim} \operatorname{Im} A_{x}=t$ off an isolated set of points.

Now let $p_{1}, \cdots, p_{r}$ be a local holomorphic basis for $E$. Then each $A\left(p_{i}\right)$ is a local holomorphic section of $F$ and for each $\left\{i_{1}, \cdots, i_{t}\right\} \subset\{1, \cdots, r\}$, $\wedge_{j=1}^{t} A\left(p_{i}\right)$ is a local holomorphic section of $\wedge^{t} F$. Since $\wedge^{t} A$ has only isolated zeros, there exists $\left\{i_{1}, \cdots, i_{t}\right\}$ such that $\bigwedge_{j=1}^{t} A\left(p_{i_{j}}\right)$ has only isolated zeros.

Let $z_{0}$ be such a zero. Then we have

$$
\bigwedge_{j=1}^{t} A\left(p_{i_{j}}\right)=\left(z-z_{0}\right)^{k} W
$$

where $W$ is a local holomorphic section of $\wedge^{t} F$ such that $W\left(z_{0}\right) \neq 0$ and $W(z)$ is decomposible for all $z$ near $z_{0}$. 
Thus $W$ defines a local rank $t$ holomorphic subbundle which coincides with $\operatorname{Im} A$ off $z_{0}$. In this way $\operatorname{Im} A$ extends to a well-defined holomorphic subbundle $\underline{\beta}$ of $F$. $\underline{\alpha}$ is constructed similarly.

Remark. Of course, a similar result holds with 'holomorphic' replaced by 'antiholomorphic' throughout.

Notation. Given $\mathscr{A}=A \otimes d z^{k}$ as in (2.2) we shall denote by $\operatorname{ker} A$ and $\operatorname{Im} A$ the holomorphic subbundles constructed in (2.2) that coincide with $\operatorname{ker} \bar{A}$ and Im $A$ almost everywhere.

B. Now let $\phi: M^{2} \rightarrow G_{k, n}$ be harmonic. We apply (2.2) to $\mathscr{A}_{\phi}^{\prime}=d z \otimes A_{\phi}^{\prime}$ to get $\underline{\operatorname{Im}} A_{\phi}^{\prime}$, a holomorphic subbundle of $\underline{\phi}^{\perp}$. We call $\underline{\operatorname{Im}} A_{\phi}^{\prime}$ the $\partial^{\prime}$-Gauss bundle of $\phi$ and denote it by $G^{\prime}(\phi)$. (Recall that $G^{\prime}(\phi)$ coincides a.e. with the image of $\partial \phi^{(1,0)}(\partial / \partial z)$, thought of as a local section of $L\left(\underline{\phi}, \underline{\phi}^{\perp}\right)$.)

Similarly we call $\operatorname{Im} A_{\phi}^{\prime \prime}$ the $\partial^{\prime \prime}$-Gauss bundle of $\phi$ and denote it by $G^{\prime \prime}(\phi)$. $G^{\prime \prime}(\phi)$ is an antiholomorphic subbundle of $\phi^{\perp}$.

Proposition 2.3. If $\phi: M^{2} \rightarrow G_{k, n}$ is harmonic, then the Gauss bundles $G^{\prime}(\phi)$ and $G^{\prime \prime}(\phi)$ are harmonic. Further $G^{\prime \prime}\left(G^{\prime}(\phi)\right) \subset \underline{\phi}$ and $G^{\prime}\left(G^{\prime \prime}(\phi)\right) \subset \underline{\phi}$

Proof. Let $\underline{R}=\left(\underline{\phi}+G^{\prime}(\phi)\right)^{\perp}$. Since $G^{\prime}(\phi)$ is a holomorphic subbundle of $\underline{\phi}^{\perp}=G^{\prime}(\phi)+\underline{R}$ we have $A_{G^{\prime}(\phi), R}^{\prime \prime}=0$ whence $A_{R, G^{\prime}(\phi)}^{\prime}$ vanishes. Further, since Im $A_{\phi}^{\prime} \subset G^{\prime}(\phi)$ by definition, $A_{\phi, R}^{\prime}$ vanishes so that the result follows at once from Lemma 2.1. Lastly, from the vanishing of $A_{G^{\prime}(\phi), R}^{\prime \prime}$, we see that $\operatorname{Im} A_{G^{\prime}(\phi)}^{\prime \prime}$ $\subset \underline{\phi}$ whence $G^{\prime \prime}\left(G^{\prime}(\phi)\right) \subset \underline{\phi}$.

The proof for $G^{\prime \prime}(\phi)$ is similar, or follows from the above by reversing the orientation on $M^{2}$.

Remark. It is easily seen that $G^{\prime \prime}\left(G^{\prime}(\phi)\right)=\underline{\phi}$ if and only if $A_{\phi}^{\prime}$ has maximal rank off an isolated set of points. Similarly $G^{\prime}\left(G^{\prime \prime}(\phi)\right)=\phi$ if and only if $A_{\phi}^{\prime \prime}$ has maximal rank. We say that $\phi$ is a $\partial^{\prime}$-(resp. $\left.\partial^{\prime \prime}\right)$-irreducible if and only if $A_{\phi}^{\prime}$ (resp. $A_{\phi}^{\prime \prime}$ ) has maximal rank (cf. §3).

C. Loosely speaking, our technique for producing new harmonic maps is the following: Let $\underline{\phi}$ be harmonic and $\underline{\alpha} \subset \underline{\phi}$ a suitable holomorphic subbundle of $\underline{\phi}$.

We then construct a new harmonic map $\underline{\dot{\phi}}$ given by

$$
\underline{\tilde{\phi}}=\left(\underline{\phi} \cap \underline{\alpha}^{\perp}\right)+\underline{\operatorname{Im}}\left(A_{\phi}^{\prime} \mid \underline{\alpha}\right)
$$

Thus we 'replace $\underline{\alpha}$ by its $\partial$ '-Gauss image'. We call this procedure 'forward replacement of $\alpha$ '. There is of course a dual notion of backward replacement of an antiholomorphic subbundle of $\phi$ by its $\partial^{\prime \prime}$-Gauss image.

We formalise these notions in the next

Theorem 2.4. Let $\underline{\phi}$ be a harmonic subbundle of $\underline{\mathbf{C}}^{n}$. 
(i) Let $\underline{\alpha}$ be a holomorphic subbundle of $\underline{\phi}$ such that $\underline{\alpha} \subset \operatorname{ker}{A_{\phi^{\perp}}^{\prime}}^{\prime} A_{\phi}^{\prime}$. Then, denoting $\underline{\operatorname{Im}}\left(A_{\phi}^{\prime} \mid \underline{\alpha}\right)$ by $G_{\phi}^{\prime}(\alpha)$, the bundle $\underline{\tilde{\phi}}$ given by

$$
\underline{\tilde{\phi}}=\underline{\phi} \cap \underline{\alpha}^{\perp}+G_{\phi}^{\prime}(\alpha)
$$

is harmonic.

Dually:

(ii) Let $\underline{\beta}$ be an antiholomorphic subbundle of $\underline{\phi}$ such that $\underline{\beta} \subset \operatorname{ker} A_{\phi^{\perp}}^{\prime \prime} \circ A_{\phi}^{\prime \prime}$. Then, denoting $\underline{\operatorname{Im}}\left(A_{\phi}^{\prime \prime} \mid \underline{\beta}\right)$ by $G_{\phi}^{\prime \prime}(\beta)$, the bundle $\underline{\underline{\phi}}$ given by

$$
\underline{\tilde{\phi}}=\underline{\phi} \cap \underline{\beta}^{\perp}+G_{\phi}^{\prime \prime}(\beta)
$$

is harmonic.

.In case (i) we say that $\tilde{\phi}$ is obtained from $\phi$ by forward replacement of $\alpha$ and in case (ii) by backward replacement of $\beta$.

Proof. The proof of (ii) will follow from the proof of (i) by reversing the orientation on $M^{2}$.

For (i), let $\underline{\alpha} \subset \underline{\phi}$ be a holomorphic subbundle contained in $\operatorname{ker} A_{\phi^{\perp}}^{\prime} \circ A_{\phi}^{\prime}$. First we observe that since $A_{\phi}^{\prime}$ is holomorphic, it follows from Proposition 1.2(i) that $A_{\alpha, \phi^{\perp}}^{\prime}=A_{\phi}^{\prime} \mid \underline{\alpha}: \underline{\alpha} \rightarrow \underline{\phi}^{\perp}$ is holomorphic so that we may apply Proposition 2.2 to construct $G_{\phi}^{\prime}(\alpha)$ which is holomorphic in $\underline{\phi}^{\perp}$. Clearly we have $G_{\phi}^{\prime}(\alpha) \subset G^{\prime}(\phi)$ and by hypothesis $A_{\phi^{\perp}}^{\prime}\left(G_{\phi}^{\prime}(\alpha)\right)=0$.

Putting $R=\left(\phi+G^{\prime}(\phi)\right)^{\perp}$ we summarize the situation in the following diagram for $\underline{\mathbf{C}}^{n}=\underline{\alpha}(1)\left(\underline{\phi} \cap \underline{\alpha}^{\perp}\right)$ (1) $G_{\phi}^{\prime}(\alpha)(1)\left(G_{\phi}^{\prime}(\alpha) \perp \cap G^{\prime}(\phi)\right)(\perp)$.

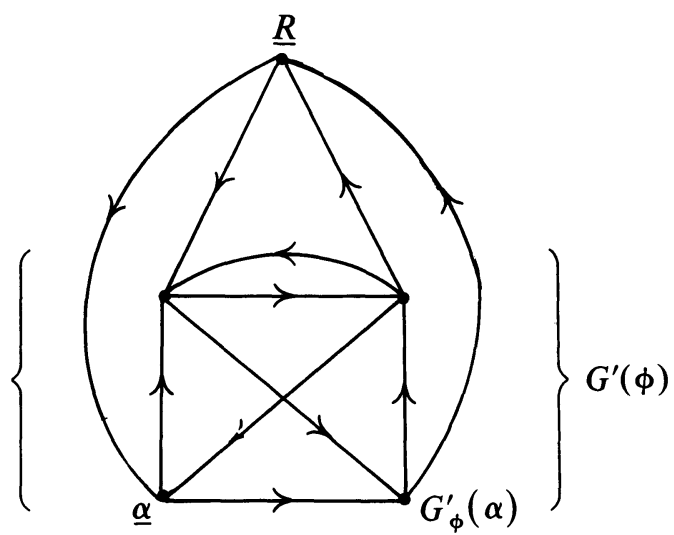

Here $A_{\alpha^{\perp} \cap \phi, \alpha}^{\prime}, A_{G_{\phi}^{\prime}(\alpha) \cap G^{\prime}(\phi), G_{\phi}^{\prime}(\alpha)}^{\prime}$ vanish since $\underline{\alpha}, G_{\phi}^{\prime}(\alpha)$ are holomorphic subbundles of $\underline{\phi}, G^{\prime}(\phi)$ respectively, $A_{G_{\phi}^{\prime}(\alpha), \phi}^{\prime}$ vanishes since $A_{G_{\phi}^{\prime}(\alpha), \phi}^{\prime}=A_{\phi^{\perp}}^{\prime}$ $\mid G_{\phi}^{\prime}(\alpha), A_{\phi, R}^{\prime}$ vanishes since $\operatorname{Im} A_{\phi}^{\prime} \subset G^{\prime}(\phi) \perp \underline{R}$ and $A_{R, G^{\prime}(\phi)}^{\prime}$ vanishes since $G^{\prime}(\phi)$ is holomorphic in $\phi^{\perp}$. 
Now put $\underline{R}_{1}=\left(\underline{\phi}+G_{\phi}^{\prime}(\alpha)\right)^{\perp}$. Then by inspection of the above diagram (1) we see that the diagram for $\underline{\mathrm{C}}^{n}=\underline{\phi}(1) G_{\phi}^{\prime}(\alpha)(1) \underline{R}_{1}$ is

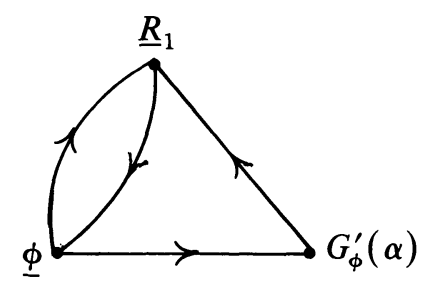

whence $\underline{R}_{1}$ is harmonic by Lemma 2.1 .

Now put $\tilde{\phi}=\phi \cap \underline{\alpha}^{\perp}+G_{\phi}^{\prime}(\alpha)$. Then, again by inspection of (1) we see that the diagram for $\underline{\mathbf{C}}^{n}=\underline{R}_{1}$ (1) $\underline{\tilde{\phi}}(1) \underline{\alpha}$ is

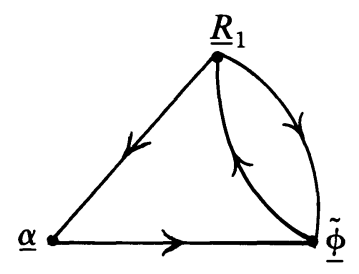

whence again by Lemma $2.1 \underline{\tilde{\phi}}$ is harmonic since $\underline{R}_{1}$ is.

Remarks. (a) Recall that a map $\phi:(M, g) \rightarrow(N, h)$ of Riemannian manifolds is weakly conformal if $\phi^{*} h=\lambda g$, where $\lambda$ is a nonnegative function on $M$. In case of a map $\phi: M^{2} \rightarrow G_{k, n}$ this condition is equivalent to

$$
\operatorname{Trace}\left(A_{\phi^{\perp}}^{\prime} \circ A_{\phi}^{\prime}\right)=0 \text {. }
$$

We say that $\phi$ is strongly conformal if the $\partial^{\prime}$ - and $\partial^{\prime \prime}$-Gauss bundles are orthogonal:

or equivalently if

$$
G^{\prime}(\phi) \perp G^{\prime \prime}(\phi)
$$

$$
A_{\phi^{\perp}}^{\prime} \circ A_{\phi}^{\prime} \equiv 0
$$

Thus strong conformality implies weak conformality and it is clear that the notions coincide if $k=1$.

Clearly, if $\phi$ is strongly conformal, we may forward replace any holomorphic subbundle of $\underline{\phi}$ to obtain a new harmonic map. Similarly backward replacement of any antiholomorphic subbundle also produces a harmonic map.

(b) If $M^{2}=S^{2}$, from Proposition 1.9 we have that $A_{\phi^{\perp}}^{\prime} \circ A_{\phi}^{\prime}$ is nilpotent and thus has nonzero kernel. This ensures the existence of holomorphic subbundles of $\phi$ to which foward replacement can be applied.

(c) With some more work Theorem 2.4 can be improved as follows: If $\underline{\alpha}$ is a holomorphic subbundle of $\underline{\phi}$ such that $A_{\phi^{\perp}}^{\prime} \circ A_{\phi}^{\prime}(\underline{\alpha}) \subset \underline{\alpha}$, then forward replacement of $\underline{\alpha}$ yields a harmonic map. Details will appear elsewhere. 
Forward and backward replacement are generically inverse to each other as the following proposition demonstrates:

Proposition 2.5. Let $\phi$ be harmonic and let $\underline{\alpha} \subset \operatorname{ker}{A_{\phi^{\perp}}^{\prime}}^{\circ} A_{\phi}^{\prime}$ be a holomorphic subbundle of $\underline{\phi}$. Let $\underline{\tilde{\phi}}=\underline{\alpha}^{\perp} \cap \underline{\phi}+G_{\phi}^{\prime}(\alpha)$ be obtained from $\underline{\phi}$ by forward replacement of $\underline{\alpha}$. Then, $\overline{G_{\phi}^{\prime}}(\alpha)$ is an antiholomorphic subbundle of $\overline{\tilde{\phi}}, G_{\phi}^{\prime}(\alpha) \subset$ $\operatorname{ker} A_{\tilde{\phi}^{\perp}}^{\prime \prime} \circ A_{\tilde{\phi}}^{\prime \prime}$ and if $\operatorname{rank} \underline{\alpha}=\operatorname{rank} G_{\phi}^{\prime}(\alpha)$, then $\underline{\phi}$ is obtained from $\underline{\dot{\phi}}$ by backward replacement of $G_{\phi}^{\prime}(\alpha)$, i.e.

$$
\underline{\phi}=G_{\phi}^{\prime}(\alpha)^{\perp} \cap \underline{\tilde{\phi}}+G_{\tilde{\phi}}^{\prime \prime}\left(G_{\phi}^{\prime}(\alpha)\right) .
$$

The corresponding statement for backward replacement of $\underline{\beta} \subset \phi$ holds also.

-Proof. We see from diagram (1) in the proof of Theorem 2.4 that $A_{G_{\phi}^{\prime}(\alpha), \alpha^{\perp} \cap \phi}^{\prime}$ vanishes so that $G_{\phi}^{\prime}(\alpha)$ is an antiholomorphic subbundle of $\tilde{\phi}$.

Further $A_{\hat{\phi}}^{\prime \prime} \mid G_{\phi}^{\prime}(\alpha)=A_{G_{\phi}^{\prime}(\alpha), \alpha}^{\prime \prime}+A_{G_{\phi}^{\prime}(\alpha), R_{1}}^{\prime \prime}$ and $A_{G_{\phi}^{\prime}(\alpha), R_{1}}^{\prime \prime}$ vanishes since from diagram (2) its adjoint $-A_{R_{1}, G_{\phi}^{\prime}(\alpha)}^{\prime}$ vanishes. Thus $A_{\dot{\phi}}^{\prime \prime} \mid G_{\phi}^{\prime}(\alpha) \subset \underline{\alpha}$. Now $A_{\tilde{\phi}^{1}}^{\prime \prime} \mid \underline{\alpha}=$ $A_{\alpha, \tilde{\phi}}^{\prime \prime}=A_{\alpha, \alpha^{\perp} \cap \phi}^{\prime \prime}+A_{\alpha, G_{\phi}^{\prime}(\alpha)}^{\prime \prime}$ and both these last summands vanish since their adjoints do by inspection of diagram (1). Thus we conclude that $G_{\phi}^{\prime}(\alpha) \subset$ $\operatorname{ker} A_{\bar{\phi}^{1}}^{\prime \prime} \circ A_{\bar{\phi}}^{\prime \prime}$ so that $G_{\phi}^{\prime}(\alpha)$ is a candidate for backwards replacement. The rank assumption ensures that the $\partial^{\prime \prime}$-Gauss image of $G_{\phi}^{\prime}(\alpha)$ is $\underline{\alpha}$ and since $G_{\phi}^{\prime}(\alpha)^{\perp}$ $\cap \tilde{\phi}=\underline{\alpha}^{\perp} \cap \underline{\phi}$ we see that this replacement produces $\underline{\phi}$.

Note. The rank condition on $\alpha$ is always satisfied if $\phi$ is $\partial^{\prime}$-irreducible.

D. We conclude this section with a short proof of the classification theorem for harmonic maps from $S^{2} \rightarrow \mathbf{C} P^{n-1}$ (see [3], [9], [17], [20]) using the methods of $\S \S 1$ and 2.

For convenience, we reverse the orientation of $S^{2}$ at the start of the proof. This interchanges holomorphic and antiholomorphic quantities.

Let $\phi: S^{2} \rightarrow \mathbf{C} P^{n-1}=G_{1, n}$ be a harmonic map. Then $A_{\phi^{\perp}}^{\prime} \circ A_{\phi}^{\prime}: \underline{\phi} \rightarrow \underline{\phi}$ is nilpotent and since rank $\underline{\phi}=1$ we have $A_{\phi^{\perp}}^{\prime} \circ A_{\phi}^{\prime}=0$ and thus $G^{\prime \prime}(\phi) \perp G^{\prime}(\phi)$. (This is nothing more than the well-known fact that any harmonic map of $S^{2}$ is weakly conformal; cf. Remark (a) following Theorem 2.4.)

Thus, putting $\underline{R}=\left(\underline{\phi}+G^{\prime}(\phi)\right)^{\perp}$ we have a diagram:

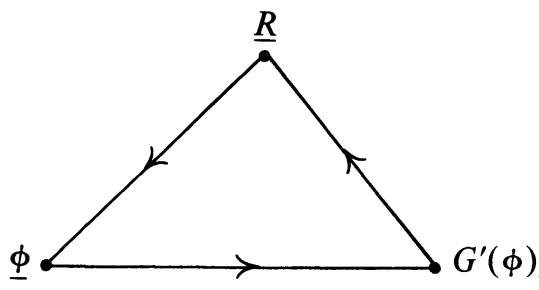


Clearly we have a holomorphic circuit and so if no edge vanishes we have $\operatorname{Im} A_{G^{\prime}(\phi), R}^{\prime} \subset \operatorname{ker} A_{R, \phi}^{\prime}$. Further since $A_{G^{\prime}(\phi)}^{\prime}$ has image contained in $\underline{R}$, we see that $G^{\prime}\left(G^{\prime}(\phi)\right)=G^{(2)}(\phi)$ is holomorphic in $\underline{R}$ from Proposition 1.2 and is contained in $\operatorname{ker} A_{R, \phi}^{\prime}$. Thus we have a diagram:

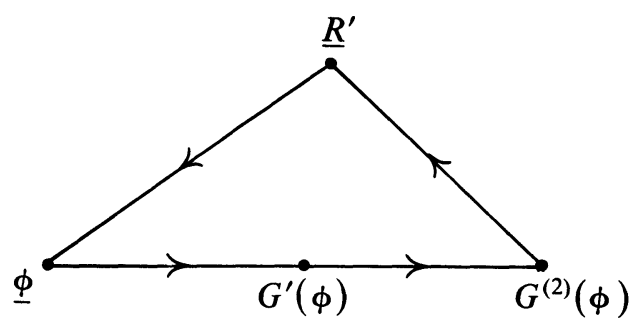

If no edge vanishes we may repeat the procedure until we have a diagram

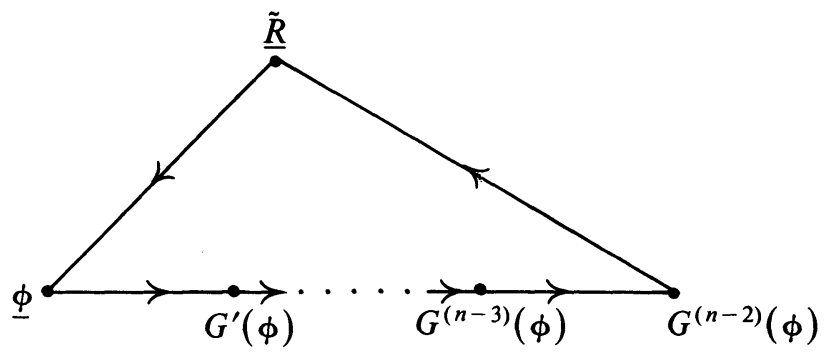

where all vertices have rank one and thus an edge must vanish. Thus for some $r \leqslant n-1$ we have a diagram

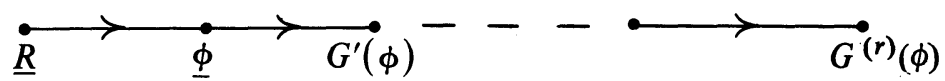

(where $R=0$ if $r=n-1)$. Thus $\underline{\psi}=G^{(r)}(\phi)$ is antiholomorphic and $\underline{\phi}=$ $G^{(-r)}(\psi)=G^{\prime \prime}\left(G^{\prime \prime} \cdots\left(G^{(r)}(\phi)\right)\right)$ or, restoring the orientation on $S^{2}, \bar{\phi}=$ $G^{(r)}(\psi)$ where $\psi: S^{2} \rightarrow \mathbf{C} P^{n-1}$ is a holomorphic map. Conversely, any such $G^{(r)}(\psi)$ is harmonic from Proposition 2.3. It is also easy to see from $G^{\prime \prime}\left(G^{\prime}(\phi)\right)$ $=\phi$ that the correspondence $(\psi, r) \leadsto G^{(r)}(\psi)$ is bijective, so adding a fullness ingredient gives

Theorem 2.6 (Eells-Wood [17], cf. Din-Zakrzewski [9], Glaser-Stora [20], Burns [3]). There is a bijective correspondence between pairs $(\psi, r)$ of full holomorphic maps $\psi: S^{2} \rightarrow \mathbf{C} P^{n-1}$ and integers $r, 0 \leqslant r \leqslant n-1$, and full 
harmonic maps $\phi: S^{2} \rightarrow \mathrm{C} P^{n-1}$ given by

$$
(\psi, r) \leadsto G^{(r)}(\psi) .
$$

3. Construction of harmonic maps $S^{2} \rightarrow G_{2, n}$

In this section we show that the techniques of forward and backward replacement can be used to construct all harmonic maps $S^{2} \rightarrow G_{2, n}$ from a very small class of harmonic maps essentially composed of holomorphic and antiholomorphic maps into $G_{2, n}$ or $\mathbf{C} P^{n-1}$.

A. Let us first introduce some terminology.

Definition. Let $\phi: M^{2} \rightarrow G_{k, n}$ be harmonic. Denote by $G^{(r)}(\phi)$ the $r$ th $\partial^{\prime}$-Gauss bundle of $\phi$ defined by

$$
G^{(1)}(\phi)=G^{\prime}(\phi), \quad G^{(r+1)}(\phi)=G^{\prime}\left(G^{(r)}(\phi)\right) .
$$

Similarly we define the $r$ th $\partial^{\prime \prime}$-Gauss bundle $G^{(-r)}(\phi)$ by

$$
G^{(-1)}(\phi)=G^{\prime \prime}(\phi), \quad G^{(-r-1)}(\phi)=G^{\prime \prime}\left(G^{(-r)}(\phi)\right) .
$$

For convenience put $G^{(0)}(\phi)=\underline{\phi}$. $\phi$ is said to have isotropy order $\geqslant r$ if $\phi \perp G^{(i)}(\phi)$ for $1 \leqslant i \leqslant r$. Of course, any harmonic map has isotropy order $\geqslant$ $\overline{1}$.

Lemma 3.1. If $\phi: M^{2} \rightarrow G_{k, n}$ has isotropy order $\geqslant r$, then

$$
G^{(i)}(\phi) \perp G^{(j)}(\phi) \text { for all } i, j \text { such that } 0<|i-j| \leqslant r \text {. }
$$

Proof. The proof is a straightforward but lengthy induction using

$$
\begin{aligned}
& \partial^{\prime} C^{\infty}\left(G^{(i)}(\phi)\right) \subset C^{\infty}\left(G^{(i)}(\phi)+G^{(i+1)}(\phi)\right), \\
& \partial^{\prime \prime} C^{\infty}\left(G^{(i)}(\phi)\right) \subset C^{\infty}\left(G^{(i)}(\phi)+G^{(i-1)}(\phi)\right),
\end{aligned}
$$

which follows from Proposition 2.3, and is left to the reader.

Definition. A harmonic map $\phi: M^{2} \rightarrow G_{k, n}$ is said to be $\partial^{\prime}$-irreducible if $\operatorname{rank} \underline{\phi}=\operatorname{rank} G^{\prime}(\phi)$ and $\partial^{\prime}$-reducible otherwise.

Similarly $\phi$ is $\partial^{\prime \prime}$-irreducible if rank $\underline{\phi}=\operatorname{rank} G^{\prime \prime}(\phi)$ and $\partial^{\prime \prime}$-reducible otherwise.

Clearly $\phi$ is $\partial^{\prime}-\left(\right.$ resp. $\partial^{\prime \prime}-$ ) irreducible if and only if $A_{\phi}^{\prime}$ (resp. $A_{\phi}^{\prime \prime}$ ) has maximal rank off an isolated set of points.

Lemma 3.2. Let $\phi: M^{2} \rightarrow G_{k, n}$ have isotropy order $\geqslant r$. If $r>n$, then for any $i$, at least one of $G^{(i)}(\phi), G^{(i+1)}(\phi), \cdots, G^{(i+r)}(\phi)$ must vanish. In particular there is a number $r_{0}$ such that if $r \geqslant r_{0}$, then at least one of $\phi, \cdots, G^{(r-1)}(\phi)$ is $\partial^{\prime}$-reducible.

Proof. By Lemma 3.1, for any $i$, the Gauss bundles $G^{(i)}(\phi), \cdots, G^{(i+r)}(\phi)$ are mutually orthogonal and thus the sum of their ranks cannot exceed rank $\underline{\mathrm{C}}^{n}=n$. q.e.d. 
Now let $h: M^{2} \rightarrow \mathbf{C} P^{n-1}$ be a holomorphic map. From $\S 2 \mathrm{D}$ we have a diagram

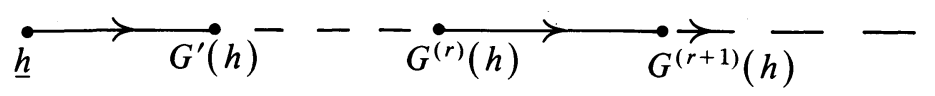

and we see that $\underline{\phi}=G^{(r)}(h) \oplus G^{(r+1)}(h)$ is a harmonic bundle. In the case that neither factor vanishes we have a harmonic map $\phi: M^{2} \rightarrow G_{2, n}$. We say that $\phi$ is a Frenet pair associated to $h$. Note that such a map is strongly isotropic in the sense of Erdem-Wood [19] (i.e., $\phi$ has isotropy order $\geqslant r$ for all $r$ ).

Compare with [10]. Further, suppose that $h, g: M^{2} \rightarrow \mathbf{C} P^{n-1}$ are holomorphic and antiholomorphic respectively and that $G^{\prime}(h) \perp g$, or equivalently $G^{\prime \prime}(g) \perp h$. Then we have a diagram

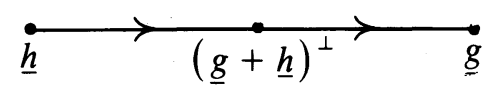

so that $(\underline{g} \oplus \underline{h})^{\perp}$ and hence $\underline{g} \oplus \underline{h}$ is harmonic. In fact $\left(\underline{h}, \underline{g}{ }^{\perp}\right)$ is a $\partial^{\prime}$-pair (see $\S 1$, examples). We say that $\underline{\phi}=\underline{g} \oplus \underline{h}$ is a mixed pair.

Clearly if $\phi$ is a mixed pair, then $\phi^{\perp}$ is strongly isotropic and in fact it can be shown that for $\phi: M^{2} \rightarrow G_{2, n}, \phi^{\perp}$ is strongly isotropic if and only if $\phi$ is \pm holomorphic or a mixed pair (c.f. §3D).

B. We are now in a position to state our main theorem of this section.

Theorem 3.3. Let $\phi: S^{2} \rightarrow G_{2, n}$ be a harmonic map. Then there is a sequence of harmonic maps $\phi_{0}, \cdots, \phi_{N}: S^{2} \rightarrow G_{2, n}$ such that

(i) $\phi_{0}$ is holomorphic, a Frenet pair associated to a holomorphic map $h$ : $S^{2} \rightarrow \mathbf{C} P^{n-1}$, or a mixed pair.

(ii) $\phi_{N}=\phi$.

(iii) For each $i<N$, there is a holomorphic line subbundle $L_{i}$ of $\underline{\phi}_{i}$ such that $\underline{\phi}_{i+1}$ is obtained from $\underline{\phi}_{i}$ by forward replacement of $L_{i}$ or backward replacement of $L_{i}^{\perp} \cap \underline{\phi}_{i}$.

Remarks. (i) Thus to construct any arbitrary harmonic $\phi: S^{2} \rightarrow G_{2, n}$ from the 'holomorphic data' $\phi_{0}$ involves the choice of a sequence of holomorphic line subbundles of rank 2 holomorphic bundles or equivalently, the choice of holomorphic sections of $\mathbf{C} P^{1}$-bundles.

(ii) In fact our proof will produce the sequence $\phi_{N}, \phi_{N-1}, \cdots, \phi_{0}$ by suitable forward and backward replacements. 
C. The proof will proceed in several steps;

First. By forward replacement, if necessary, we produce from $\phi$ a $\partial^{\prime}$ reducible map.

Then. By backward replacement applied to the $\partial^{\prime}$-reducible map we produce a Frenet pair.

Holomorphic maps into $G_{2, n}$ or mixed pairs will appear when these procedures degenerate.

Remark. It is an irritating feature of all twistor constructions that to produce holomorphic data one must work with antiholomorphic second fundamental forms $A_{\phi}^{\prime \prime}$. We remedy this by reversing the orientation of $S^{2}$ at the beginning of the proof, working with holomorphic second fundamental forms and then restoring the original orientation to complete the proof.

Lemma 3.2 shows that if $\phi: S^{2} \rightarrow G_{k, n}$ is harmonic and of sufficiently high isotropy order, then either $\phi$ or one of its $\partial^{\prime}$-Gauss maps is $\partial^{\prime}$-reducible. The next proposition shows how forward replacement of a suitable subbundle increases the isotropy order.

Proposition 3.4. Let $\phi: S^{2} \rightarrow G_{2, n}$ be harmonic of isotropy order $\geqslant r(r \geqslant 1)$. Suppose that $\phi, G^{\prime}(\phi), \cdots, G^{(r)}(\phi)$ are all $\partial^{\prime}$-irreducible. Then

(i) The second fundamental form $A_{G^{(r)}(\phi), \phi}^{\prime}$ is holomorphic.

(ii) Let $\underline{\alpha}$ denote $\underline{\operatorname{Im}} A_{G^{(r)}(\phi), \phi}^{\prime}$; then rank $\alpha \leqslant 1$.

(iii) If $\tilde{\phi}$ is obtained from $\phi$ by forward replacement of $\underline{\alpha}$, i.e. $\tilde{\phi}=\underline{\alpha}^{\perp} \cap \underline{\phi}+$ $G_{\phi}^{\prime}(\alpha)$, then $\tilde{\phi}: S^{2} \rightarrow G_{2, n}$ is harmonic of isotropy order $\geqslant r+1$.

Remarks. (i) If $\alpha=0, \phi=\tilde{\phi}$ already has isotropy order $\geqslant r+1$.

(ii) By Proposition 2.5, if $\underline{\alpha} \neq 0$, then we recover $\phi$ from $\tilde{\phi}$ by backwards replacement of $G_{\phi}^{\prime}(\alpha)$ which is an antiholomorphic line subbundle of $\tilde{\phi}$.

Proof. For $r=1, A_{G^{\prime}(\phi), \phi}^{\prime}=A_{\phi^{\perp}}^{\prime} \mid G^{\prime}(\phi)$ which is holomorphic by Proposition 1.2(i) since $A_{\phi^{\perp}}^{\prime}$ is holomorphic. Now $A_{G^{\prime}(\phi), \phi}^{\prime} A_{\phi, G^{\prime}(\phi)}^{\prime}: \underline{\phi} \rightarrow \underline{\phi}$ is a holomorphic circuit and is therefore not an isomorphism. Since $\overline{A_{\phi, G^{\prime}(\phi)}^{\prime}}$ is an isomorphism by hypothesis we have rank $\alpha \leqslant 1$, which establishes (i) and (ii) for $r=1$.

Now for $r \geqslant 1$ we have a diagram

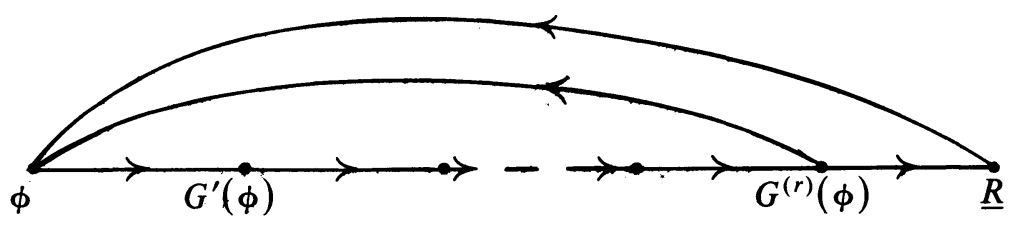

where $\underline{R}=\left(\underline{\phi} \oplus \cdots \oplus G^{(r)}(\phi)\right)^{\perp}$. Note that $\underline{R} \neq 0$ since $G^{(r)}(\phi)$ is $\partial^{\prime}-$ irreducible. By the above, and using Proposition 1.5 for $r>1$, we see that the 
inner circuit $\left\{\underline{\phi}, \cdots, G^{(r)}(\phi), \underline{\phi}\right\}$ is holomorphic with all vertices of rank 2 . Thus by Proposition 1.8, since all $A_{G^{(i)}(\phi), G^{(i+1)}(\phi)}^{\prime}$ are isomorphisms for $0 \leqslant i$ $\leqslant r-1$ we have that $\operatorname{Im} A_{G^{(r)}(\phi), \phi}^{\prime}$ has dimension $\leqslant 1$ and so rank $\underline{\alpha} \leqslant 1$. This establishes (i) and (ii).

Now observe that, as already remarked, if $\underline{\alpha}$ and hence $A_{G^{(r)}(\phi), \phi}^{\prime}$ vanishes, $\operatorname{Im} A_{G^{(r)}(\phi)}^{\prime} \subset \underline{R}$ whence $G^{(r+1)}(\phi) \perp \underline{\phi}$ and $\phi$ has isotropy order $\geqslant r+1$.

So assume that rank $\underline{\alpha}=1$. We must check that $\underline{\alpha}$ is a candidate for forward replacement, i.e., that $\underline{\alpha} \subset \operatorname{ker} A_{\phi^{1}}^{\prime} \circ A_{\phi}^{\prime}$. For $r>1, A_{\phi^{1}}^{\prime} \circ A_{\phi}^{\prime}$ vanishes and there is nothing to prove. For $r=1$, since $A_{\phi^{1}}^{\prime} \circ A_{\phi}^{\prime}$ is nilpotent, we have $\left(A_{\phi^{\perp}}^{\prime} \circ A_{\phi}^{\prime}\right)^{2}=0$ and since $\underline{\alpha}$ coincides with $\operatorname{Im} A_{\phi^{\perp}}^{\prime} \circ A_{\phi}^{\prime}$ off an isolated set of points we see that $\underline{\alpha} \subset \operatorname{ker} A_{\phi^{\perp}}^{\prime} \circ A_{\phi}^{\prime}$.

Now $A_{\phi, G^{\prime}(\phi)}^{\prime} \mid \underline{\alpha}$ is holomorphic by Proposition 1.2(i) so let $\underline{\alpha}_{1}$ be the holomorphic subbundle of $G^{\prime}(\phi)$ defined by its image. Similarly, define $\underline{\boldsymbol{\alpha}}_{i} \subset G^{(i)}(\phi)$ by

$$
\underline{\operatorname{Im}}\left(A_{G^{(i-1)}(\phi), G^{(i)}(\phi)}^{\prime} \mid \underline{\alpha}_{i-1}\right)=\underline{\alpha}_{i} \quad \text { for } 1 \leqslant i \leqslant r .
$$

Also, let $\underline{\beta}_{i}=\underline{\alpha}_{i}^{\perp} \cap G^{(i)}(\phi)$ for $1 \leqslant i \leqslant r$.

By Proposition 1.2, the restrictions of the $A_{G^{(i)}(\phi), G^{(i+1)}(\phi)}^{\prime}$ and $A_{G^{(r)}(\phi), \phi}^{\prime}$ to $\underline{\boldsymbol{\alpha}}, \underline{\boldsymbol{\alpha}}_{1}, \cdots, \underline{\boldsymbol{\alpha}}_{r}$ are holomorphic and thus we have a holomorphic circuit $\left\{\underline{\alpha}_{1}, \underline{\alpha}_{1}, \cdots, \underline{\alpha}_{r}, \underline{\alpha}\right\}$ with all vertices of rank 1 , and so the composition vanishes. Since each $A_{G^{(i)}(\phi), G^{(i+1)}(\phi)}^{\prime}$ is an isomorphism we conclude that $\underline{\alpha}_{r}$ $=\operatorname{ker} A_{G^{(r)}(\phi), \phi}^{\prime}$.

Thus we have a diagram

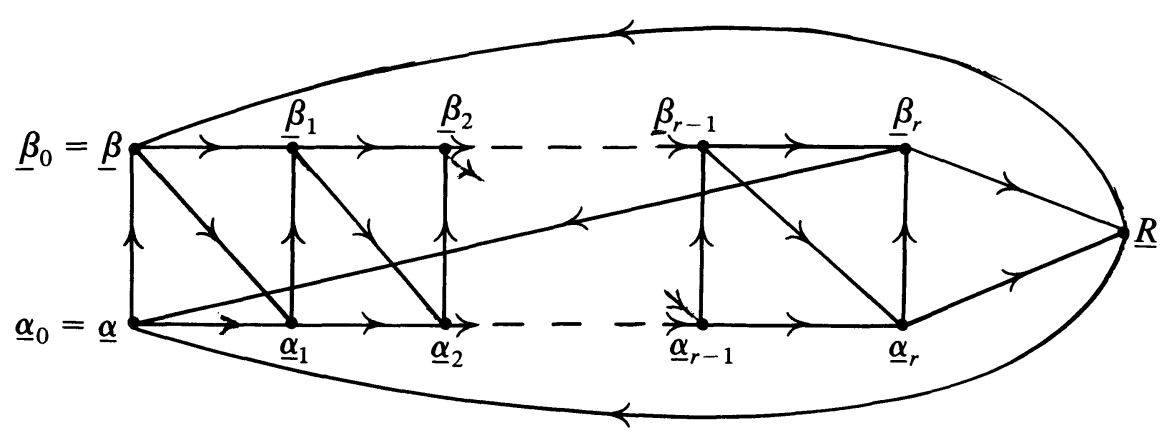

Inspection of the diagram shows that each $A_{\alpha_{i}, \alpha_{i+1}}^{\prime}, A_{\beta_{i}, \beta_{i+1}}^{\prime}, 0 \leqslant i \leqslant r-1$, is holomorphic as is $A_{\beta_{r}, \alpha_{0}}^{\prime}$ and $A_{\alpha_{r}, R}^{\prime}$, by Proposition 1.5. Further $A_{\alpha_{r}, R}^{\prime}=$ $A_{G^{(r)}(\phi)}^{\prime} \mid \underline{\alpha}_{r}$ is nonzero since $G^{(r)}(\phi)$ is $\partial^{\prime}$-irreducible, so let $\underline{\alpha}_{r+1}$ denote $\underline{\operatorname{Im}} A_{\alpha_{r}, R}^{\prime}$ 
which is a holomorphic subbundle of $\underline{R}$ and let $\underline{\beta}_{r+1}=\underline{\alpha}_{r+1}^{\perp} \cap \underline{R}$. Note that rank $\underline{\beta}_{r+1}$ need not be 1 in general but all other $\underline{\alpha}_{i}, \underline{\beta}_{i}$ have rank 1 .

Thus we have:

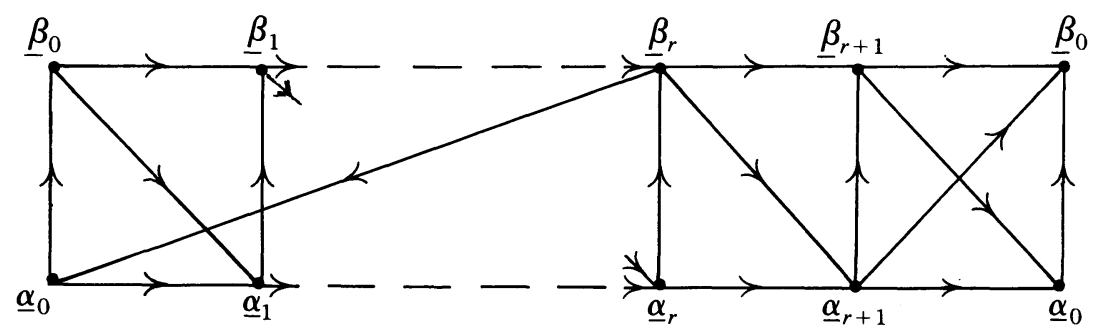

where the extreme vertical edges are identified. By inspection and Proposition 1.5 we see that $A_{\alpha_{r+1}, \beta_{0}}^{\prime}$ is holomorphic and we have a holomorphic circuit $\left\{\alpha_{0}, \cdots, \alpha_{r+1}, \beta_{0}, \cdots, \beta_{r}, \alpha_{0}\right\}$, the composition of which must vanish. Thus $A_{\alpha_{r+1}, \beta_{0}}^{\prime}$ vanishes since all other edges are nonzero being the restrictions of the various $A_{G^{(i)}(\phi)}^{\prime}$ or the restriction of $A_{G^{(r)}(\phi), \phi}^{\prime}$ to the complement of its kernel.

Now replace $\alpha_{0}$ by its $\partial^{\prime}$-Gauss image to obtain $\tilde{\phi}=\underline{\beta}_{0}+\underline{\alpha}_{1}$. From the diagram we see that

$$
G^{(i)}(\tilde{\phi})=\underline{\beta}_{i}+\underline{\alpha}_{i+1}, \quad i=1, \cdots, r,
$$

and since $A_{\alpha_{r+1}, \beta_{0}}^{\prime}=0$ we see that $G^{(r+1)}(\tilde{\phi}) \subset \underline{\beta}_{r+1}+\underline{\alpha}_{0}$.

Thus $G^{(i)}(\tilde{\phi}) \perp \tilde{\phi}$ for $1 \leqslant i \leqslant r+1$ and so $\phi$ has isotropy order $\geqslant r+1$. This concludes the proof.

Thus combining Lemma 3.2 and Proposition 3.4 we see that by forward replacement of line bundles if necessary we arrive at a harmonic map $\tilde{\phi}$ which is $\partial^{\prime}$-reducible or has a $\partial^{\prime}$-reducible $i$ th Gauss bundle for some $i$. To complete the first stage of the proof we must show that a Gauss bundle of a harmonic $\phi$ may be obtained from $\phi$ by iterated forward replacement of a line subbundle.

Lemma 3.5. Let $\phi: S^{2} \rightarrow G_{2, n}$ be a harmonic map which is $\partial^{\prime}$-irreducible. Then there is a holomorphic line subbundle $\underline{\alpha}$ of $\underline{\phi}$ such that forward replacement of $\underline{\alpha}$ yields a harmonic bundle $\underline{\tilde{\phi}}$ and forward replacement of $\underline{\alpha}^{\perp} \cap \tilde{\boldsymbol{\phi}}$ yields $G^{\prime}(\phi)$.

Remark. If $\phi$ has isotropy order $\geqslant 2$ any $\underline{\alpha}$ will do, otherwise $\underline{\alpha}$ is given by $\underline{\operatorname{ker}} A_{\phi^{+}}^{\prime} \circ A_{\phi}^{\prime}$.

Proof. Let $\underline{\alpha}$ be given by $\underline{\operatorname{ker}} A_{\phi^{\perp}}^{\prime} \circ A_{\phi}^{\prime}$ if $A_{\phi^{\perp}}^{\prime} \circ A_{\phi}^{\prime}$ is nonzero and let $\underline{\alpha}$ be any holomorphic line subbundle otherwise. Then, applying the methods of 
Proposition 3.4 we have a diagram

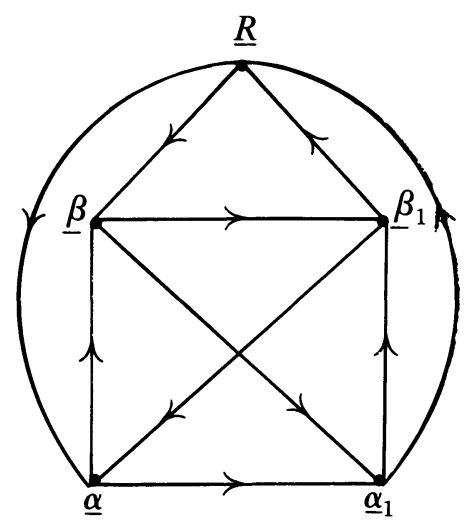

where $\underline{\beta}=\underline{\alpha}^{\perp} \cap \underline{\phi}, \underline{\alpha}_{1}=G_{\phi}^{\prime}(\alpha), \underline{\beta}_{1}=G_{\phi}^{\prime}(\alpha)^{\perp} \cap G^{\prime}(\phi)$ and $A_{\beta_{1}, \alpha}^{\prime}$ vanishes if $\phi$ has isotropy order $\geqslant 2$. We see that $\underline{\beta}$ is holomorphic in $\underline{\phi}=\underline{\beta}+\underline{\alpha}_{1}$ and $A_{\tilde{\phi}}^{\prime}(\underline{\beta}) \subset \underline{\beta}_{1}$. Further, $A_{\tilde{\phi}^{\perp}}^{\prime}\left(\underline{\beta}_{1}\right)=0$ so $\underline{\beta} \subset \operatorname{ker} A_{\tilde{\phi}^{\perp}}^{\prime} \circ A_{\tilde{\phi}^{\prime}}^{\prime}$. Thus $\underline{\beta}$ is a candidate for forward replacement and since $A_{\beta, \beta_{1}}^{\prime}$ is an isomorphism, by $\partial^{\prime}$-irreducibility of $\phi$, we see that $\underline{\beta}^{\perp} \cap \underline{\hat{\phi}}+G_{\tilde{\phi}}^{\prime}(\beta)=G^{\prime}(\phi)$ which concludes the proof.

Now observe that for all the forward replacements considered above we have $\operatorname{rank} \underline{\alpha}=\operatorname{rank} G_{\phi}^{\prime}(\alpha)$ so that we may apply Proposition 2.5 to reverse the procedure and conclude

Proposition 3.6. Let $\phi: S^{2} \rightarrow G_{2, n}$ be a harmonic map. Then there is a sequence of harmonic maps $\phi_{0}, \cdots, \phi_{N}: S^{2} \rightarrow G_{2, n}$ such that

(i) $\phi_{0}$ is $\partial^{\prime}$-reducible and is thus antiholomorphic $\left(G^{\prime}\left(\phi_{0}\right)=0\right)$ or has rank $G^{\prime}\left(\phi_{0}\right)=1$.

(ii) $\phi_{N}=\phi$.

(iii) For each $i<N$ there is a holomorphic line bundle $\underline{L}_{i}$ of $\phi_{i}$ such that $\underline{\phi}_{i+1}$ is obtained from $\underline{\phi}_{i}$ by backward replacement of $\underline{L}_{i}^{\perp} \cap \underline{\phi}_{i}$.

To finish the proof of Theorem 3.2, we must deal with nonantiholomorphic $\partial$ '-reducible maps. We distinguish two cases:

(i) We show in Proposition 3.7 that if $A_{\phi}^{\prime \prime}\left(\underline{\operatorname{ker}} A_{\phi}^{\prime \perp}\right)=0$, then $\phi$ is, up to a change of orientation, of the desired form, whereas

(ii) in Proposition 3.8 we show that if $A_{\phi}^{\prime \prime}\left(\underline{\operatorname{ker}} A_{\phi}^{\prime \perp}\right) \neq 0$, a suitable backward replacement produces a map of increased isotropy order so that by iterating the procedure we eventually get a map of type (i).

Proposition 3.7. Let $\phi: S^{2} \rightarrow G_{2, n}$ be harmonic with $G^{\prime}(\phi)$ of rank 1. If $A_{\phi}^{\prime \prime}\left(\underline{\operatorname{ker}} A_{\phi}^{\prime \prime}\right)=0$, then either (a) there is an antiholomorphic map g: $S^{2} \rightarrow \mathbf{C} P^{n-1}$ and $\bar{\phi}=G^{(-r)}(g) \oplus G^{(-r-1)}(g)$ for some integer $r \geqslant 0$, (it can be shown that $\phi$ is a Frenet pair) or (b) there are maps $\mathrm{g}, h: S^{2} \rightarrow \mathrm{C} P^{n-1}$ antiholomorphic and 
holomorphic respectively such that $G^{\prime}(h) \perp \underline{g}$ and $\underline{\phi}=\underline{g} \oplus \underline{h}$, i.e. $\underline{\phi}$ is a mixed pair.

Proof. $A_{\phi}^{\prime} \circ A_{G^{\prime}(\phi), \phi}^{\prime}: G^{\prime}(\phi) \rightarrow G^{\prime}(\phi)$ is holomorphic by Proposition 1.2 and the harmonicity of $\phi$ so $A_{\phi^{\perp}}^{\prime}\left(G^{\prime}(\phi)\right) \subset \operatorname{ker} A_{\phi}^{\prime}$. Thus denoting $\underline{\operatorname{ker}} A_{\phi}^{\prime}$ by $\underline{\alpha}$ and putting $\underline{\beta}=\underline{\alpha}^{\perp} \cap \underline{\phi}$ we have a diagram (the edge $\underline{R} \rightarrow \underline{\beta}$ vanishing by hypothesis; here $\left.R=\left(\underline{\phi}+G^{\prime}(\phi)\right)^{\perp}\right)$ :

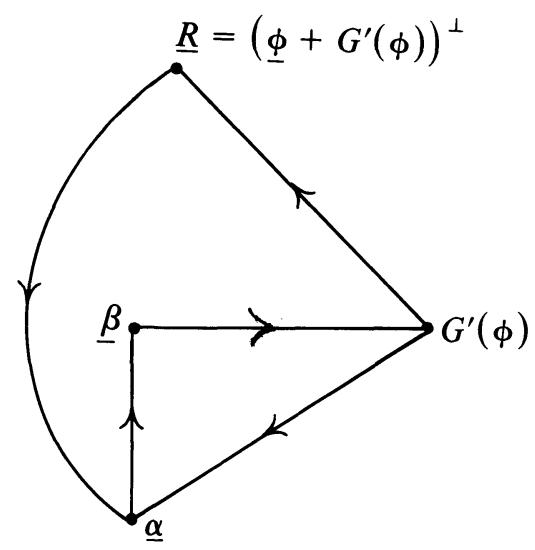

The circuit $\left\{\underline{\alpha}, \underline{\beta}, G^{\prime}(\phi), \underline{\alpha}\right\}$ is holomorphic and so at least one edge must vanish. If $A_{\alpha, \beta}^{\prime}$ vanishes we have a diagram

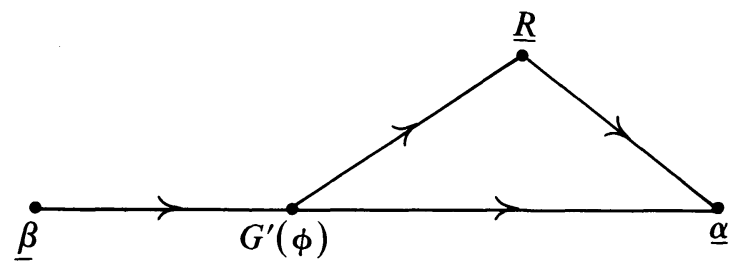

so that $\underline{\beta}=\underline{h}, \underline{\alpha}=\underline{g}$, where $h, g: S^{2} \rightarrow \mathbf{C} P^{n-1}$ are holomorphic and antiholomorphic respectively, and $G^{\prime}(h) \perp \underline{g}$.

Otherwise, since $A_{\beta, G^{\prime}(\phi)}^{\prime}$ cannot vanish without contradicting rank $G^{\prime}(\phi)=1$, $A_{G^{\prime}(\phi), \alpha}^{\prime}=0$ and we have the diagram

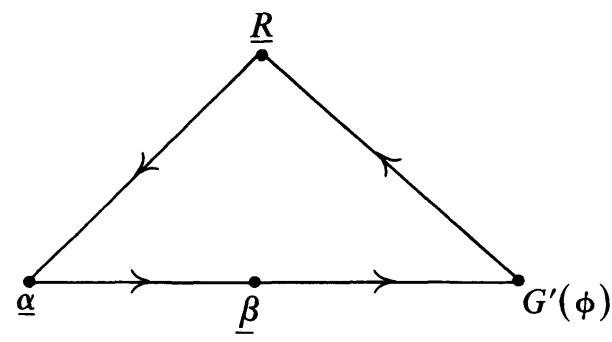


to which the argument of $\S 2 \mathrm{D}$ can be applied to conclude that $\underline{\alpha}=G^{(-r-1)}(g)$, $\underline{\beta}=G^{(-r)}(g)$ for some antiholomorphic $g: S^{2} \rightarrow \mathbf{C} P^{n-1}$.

Proposition 3.8. Let $\phi: S^{2} \rightarrow G_{2, n}$ be harmonic of isotropy order $\geqslant r \geqslant 1$ with $\operatorname{rank} G^{(i)}(\phi)=1,1 \leqslant i \leqslant r$. Suppose $A_{\phi}^{\prime \prime}\left(\operatorname{ker} A_{\phi}^{\prime \perp}\right) \neq 0$ and let $\underline{\alpha}$ be the holomorphic subbundle of $\phi$ defined by $\operatorname{ker} A_{\phi}^{\prime}$. Then backward replacement of $\underline{\beta}=\underline{\alpha}^{\perp} \cap \underline{\phi}$ produces a harmonic map $\tilde{\bar{\phi}}: S^{2} \rightarrow G_{2, n}$ of isotropy order $\geqslant r+1$ and $\operatorname{rank} \bar{G}^{(i)}(\tilde{\phi})=1,1 \leqslant i \leqslant r+1$.

Proof. We have the diagram

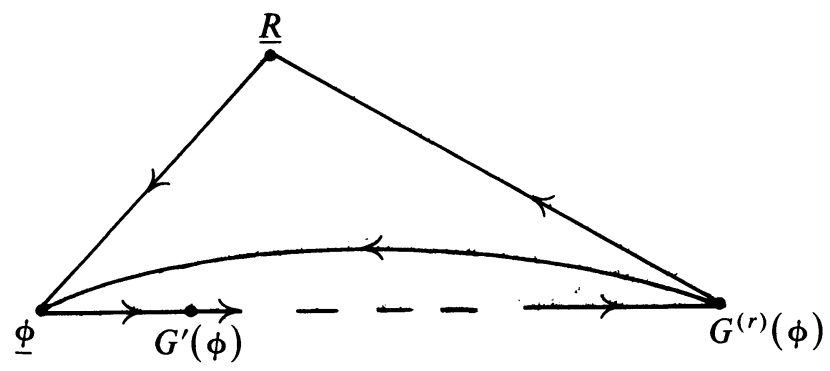

where the inner circuit is holomorphic from which we conclude that $\underline{\operatorname{Im}} A_{G^{(r)}(\phi), \phi}^{\prime} \subset \underline{\alpha}=\underline{\operatorname{ker}} A_{\phi}^{\prime}$ since all other edges are isomorphisms. Thus we have:

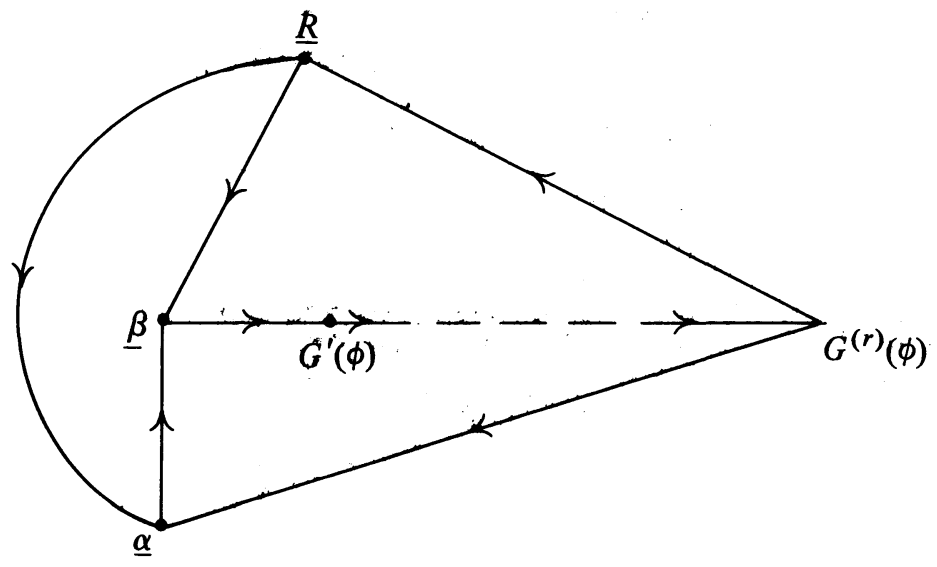

We see that $A_{\phi}^{\prime \prime}(\underline{\beta}) \subset \underline{R}$ and $A_{\phi^{\perp}}^{\prime \prime}(\underline{R})=\underline{0}$ so that $A_{\phi^{\perp}}^{\prime \prime} \circ A_{\phi}^{\prime \prime}(\underline{\beta})=\underline{0}$ whence $\underline{\beta}$ 
is a candidate for backward replacement. Thus we have

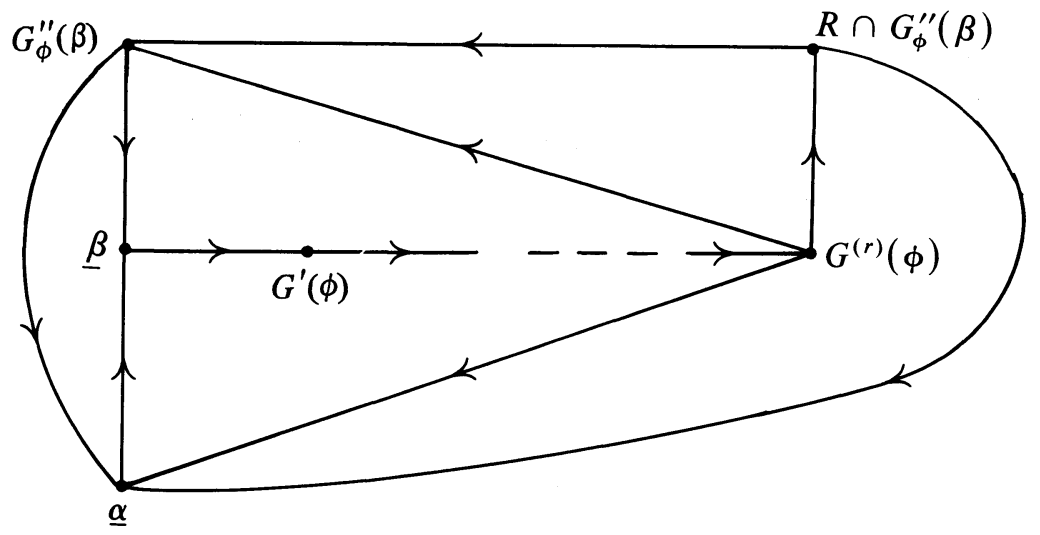

So putting $\underline{\tilde{\phi}}=\underline{\alpha}+G_{\phi}^{\prime \prime}(\beta)$ we see that $G^{\prime}(\tilde{\phi})=\underline{\beta}, G^{(i)}(\tilde{\phi})=G^{(i+1)}(\phi)$ and the proposition follows.

We now iterate until for some $\tilde{\phi}, A_{\tilde{\phi}}^{\prime \prime}\left(\underline{\operatorname{ker}} A_{\tilde{\phi}}^{\prime \perp}\right)$ vanishes (which must happen to avoid contradicting Lemma 3.2). Observing that all backward replacements in Proposition 3.8 are invertible by Lemma 3.5 the proof of Theorem 3.3 now follows by reversing the orientation on $S^{2}$ (see Remark at the beginning of this section). Note that the property of being a mixed pair is independent of orientation, while if a map satisfies conclusion (a) of Proposition 3.7, it is clear that it is a Frenet pair with respect to the conjugate complex structure on $M^{2}$.

D. We conclude this section with an application of these ideas to the construction of strongly isotropic harmonic maps of an arbitrary Riemann surface into $G_{2, n}$.

Recall that a map $\phi: M^{2} \rightarrow G_{k, n}$ is strongly isotropic if $\phi$ has isotropy order $\geqslant r$ for all $r$. Erdem and Wood showed that all strongly isotropic harmonic maps arise from $\partial^{\prime}$-pairs as in $\S 1$, i.e., there is a $\partial^{\prime}$-pair $(V, X)$ such that $\underline{\phi}=V^{\perp} \cap X$. However, in case $k=2$ we can prove a more delicate result.

Theorem 3.9. Let $M^{2}$ be a Riemann surface and $\phi: M^{2} \rightarrow G_{2, n}$ a strongly isotropic harmonic map. Then there is a sequence of strongly isotropic harmonic maps $\phi_{0}, \cdots, \phi_{N}: M^{2} \rightarrow G_{2, n}$ such that

(i) $\phi_{0}$ is holomorphic, a Frenet pair associated to a holomorphic curve $h$ : $M^{2} \rightarrow \mathrm{CP}^{n-1}$ or a mixed pair.

(ii) $\phi_{N}=\phi$.

(iii) There is a $k, 0 \leqslant k \leqslant N$, and holomorphic line subbundles $\underline{L}_{i}$ of $\underline{\phi}_{i}$ for $0 \leqslant i<k$ such that $\underline{\phi}_{i+1}$ is obtained from $\underline{\phi}_{i}$ by backward replacement of $\underline{L}_{i}^{\perp} \cap \underline{\phi}_{i}, 0 \leqslant i \leqslant k$, and for $i \geqslant k, \underline{\phi}_{i+1}=G^{\prime}\left(\bar{\phi}_{i}\right)$. 
Thus any strongly isotropic harmonic map is obtained from our 'holomorphic data' by choosing holomorphic sections of $\mathbf{C} P^{1}$ bundles and differentiating. Of course, in this context, $G^{\prime}\left(\phi_{i}\right)$ is obtained from $\underline{\phi}_{i}$ by forward replacement of $\underline{\phi}_{i}$ itself since $A_{\phi_{i}^{+}}^{\prime} \circ A_{\phi_{i}}^{\prime}$ vanishes by strong isotropy.

Proof of Theorem 3.9. The proof is based on observation that for strongly isotropic maps, the procedures in the proof of Theorem 3.3 do not require the vanishing of any holomorphic differentials and thus apply to maps of an arbitrary Riemann surface.

For completeness, we sketch the argument in this case. Let $r$ be the largest number such that $G^{(r)}(\phi) \neq 0$, finite by Lemma 3.2. Then putting $\underline{R}=$ $\left(\sum_{0}^{r} G^{(i)}(\phi)\right)^{\perp}$ and using Lemma 3.1 we have a diagram

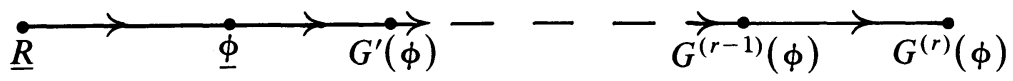

Let $i$ be the largest number such that $\operatorname{rank} G^{(i)}(\phi)=2$. Then $G^{(i)}(\phi)$ is $\partial^{\prime}$-reducible and $\phi=G^{(-i)}\left(G^{(i)}(\phi)\right)$ by Proposition 2.3. If $i=r, G^{(i)}(\phi)$ is antiholomorphic and a reversal of orientation complete the proof. Otherwise put $G^{(i)}(\phi)=\tilde{\tilde{\phi}}$ and let $s$ be the largest number such that $G^{(s)}(\tilde{\phi}) \neq 0$. Then we have a diagram

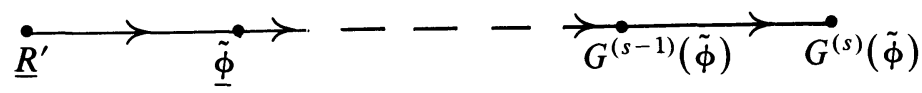

where $\operatorname{rank} G^{(i)}(\tilde{\phi})=1,1 \leqslant i \leqslant s$. Now if $A_{\tilde{\phi}}^{\prime \prime}\left(\operatorname{ker} A_{\tilde{\phi}}^{\prime}\right)^{\perp}=0$ we put $\underline{\alpha}=\underline{\operatorname{ker}} A_{\tilde{\phi}}^{\prime}$ and $\underline{\beta}=\underline{\alpha}^{\perp} \cap \underline{\phi}$ and get a diagram

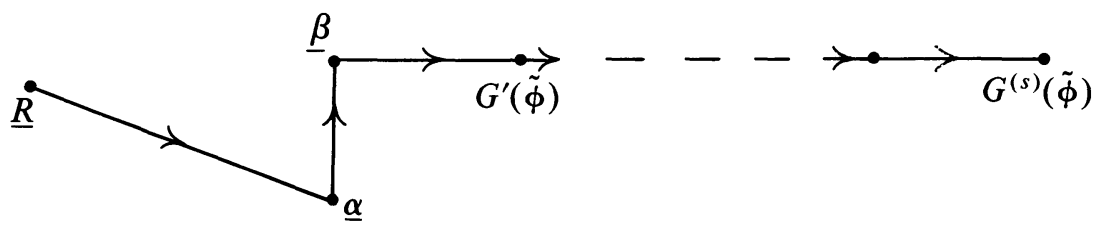

from which it is clear that $\underline{\tilde{\phi}}=\underline{\alpha} \oplus \underline{\beta}$ is either a mixed pair, if $A_{\alpha, \beta}^{\prime}$ vanishes, or $\underline{\alpha}, \underline{\beta}$ are consecutive $\partial^{\prime \prime}$-Gauss bundles of the antiholomorphic map $G^{(s)}(\tilde{\phi})$ whence after a change of orientation $\tilde{\phi}$ is a Frenet pair.

Lastly, if $A_{\tilde{\phi}}^{\prime \prime}\left(\operatorname{ker} A_{\tilde{\phi}}^{\prime \perp}\right) \neq 0$, then the backward replacement procedures of Proposition 3.8 can be applied to find a map to which the above argument may be applied. 


\section{Reducibility and harmonic maps of $S^{2}$ into $G_{k, n}, k=3,4,5$}

A. Let $\phi: M^{2} \rightarrow G_{k, n}$ be a harmonic map. Recall from $\S 3$ that $\phi$ is $\partial^{\prime}$-reducible if rank $G^{\prime}(\phi)<\operatorname{rank} \underline{\phi}$ or equivalently if $\underline{\operatorname{ker}} A_{\phi}^{\prime} \neq \underline{0}$.

The following result shows that $\partial^{\prime}$-reducible or $\partial^{\prime \prime}$-reducible harmonic maps $M^{2} \rightarrow G_{k, n}$ can be constructed from harmonic maps into Grassmannians $M^{2} \rightarrow G_{t, n}, t<k$.

Theorem 4.1 (Reduction theorem). 1.(a) Let $\phi: M^{2} \rightarrow G_{k, n}$ be harmonic and $\partial^{\prime}$-reducible. Then there is an integer $t, 0 \leqslant t \leqslant k-1$, a harmonic map $\psi$ : $M^{2} \rightarrow G_{t, n}$ and a nonzero antiholomorphic subbundle $\underline{\beta}$ of $\left(\underline{\psi}+G^{\prime}(\psi)\right)^{\perp}$ such that $\underline{\phi}=\underline{\psi} \oplus \underline{\beta}$.

(b) Conversely, given $\psi: M^{2} \rightarrow G_{t, n}$ harmonic and a nonzero antiholomorphic subbundle $\underline{\beta}$ of $\left(\underline{\psi}+G^{\prime}(\psi)\right)^{\perp}$, then putting $\underline{\phi}=\underline{\psi} \oplus \underline{\beta}$ gives a $\partial^{\prime}$-reducible harmonic map $\phi: \bar{M}^{2} \rightarrow G_{k, n}, k=t+\operatorname{rank} \underline{\beta}$.

Dually:

2.(a) Let $\phi: M^{2} \rightarrow G_{k, n}$ be harmonic and $\partial^{\prime \prime}$-reducible. Then there is an integer $t, 0 \leqslant t \leqslant k-1$, a harmonic map $\psi: M \rightarrow G_{t, n}$ and a nonzero holomorphic subbundle $\underline{\alpha}$ of $\left(\underline{\psi}+G^{\prime \prime}(\psi)\right)^{\perp}$ such that $\underline{\phi}=\underline{\psi} \oplus \underline{\alpha}$.

(b) Given $\psi: M^{2} \rightarrow G_{t, n}$ harmonic and a nonzero holomorphic subbundle $\underline{\alpha}$ of $\left(\underline{\psi}+G^{\prime \prime}(\psi)\right)^{\perp}$, then putting $\phi=\underline{\psi} \oplus \underline{\alpha}$ gives a $\partial^{\prime \prime}$-reducible harmonic map $\phi$ : $\bar{M}^{2} \rightarrow G_{k, n}$, where $k=t+\operatorname{rank} \underline{\alpha}$.

Proof. 2. will follow from 1 . by reversing the orientation.

For 1.(a) let $\underline{\beta}=\underline{\operatorname{ker}} A_{\phi}^{\prime}$, nonzero by hypothesis and put $\underline{\psi}=\underline{\beta}{ }^{\perp} \cap \underline{\phi}$. Then since $\underline{\beta}$ is a holomorphic subbundle of $\phi$ we have $A_{\psi, \beta}^{\prime}=0$ and since $A_{\phi}^{\prime} \mid \underline{\beta}$ vanishes by definition we have $A_{\beta, \phi^{\perp}}^{\prime}=0$. Thus we have a diagram

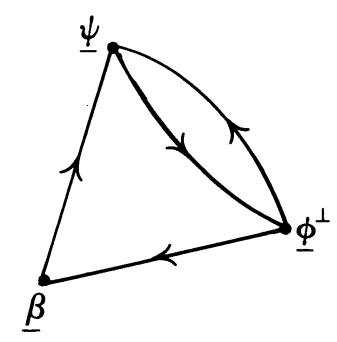

whence $\psi$ is harmonic by Lemma 2.1 since $\phi^{\perp}$ is. Further since $A_{\psi, \beta}^{\prime}$ vanishes we see that $G^{\prime}(\psi) \subset \underline{\phi}^{\perp}$ so that $\underline{\beta} \subset\left(\underline{\psi}+G^{\prime}(\psi)\right)^{\perp}$ and since $A_{\beta, \phi^{\perp}}^{\prime}$ vanishes we see that $\underline{\beta}$ is an antiholomorphic subbundle of $\underline{\beta} \oplus \underline{\phi}^{\perp}$ and so a fortiori an antiholomorphic subbundle of $\left(\psi+G^{\prime}(\psi)\right)^{\perp}$ which is contained in $\underline{\beta} \oplus \phi^{\perp}$. 
(b) For the converse let $\psi: M^{2} \rightarrow G_{t, n}$ be harmonic and put $\underline{R}=$ $\left(\psi \oplus G^{\prime}(\psi)\right)^{\perp}$. Then as usual we have a diagram:

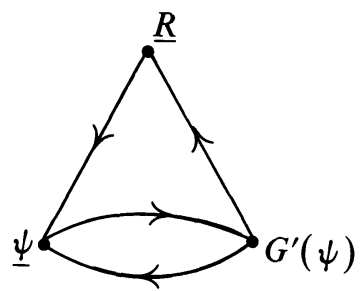

Now if $\underline{\beta}$ is an antiholomorphic subbundle of $\underline{R}$ we have $A_{\beta, \beta^{\perp} \cap R}^{\prime}=0$ and since $A_{R, G^{\prime}(\psi)}^{\prime}$ vanishes we have $A_{R \cap \beta^{\perp}, G^{\prime}(\psi)}^{\prime}=0$. Thus putting $\underline{\phi}=\underline{\psi} \oplus \underline{\beta}$ we have a diagram

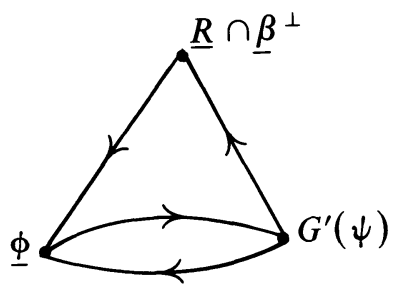

whence $\phi$ is harmonic by Lemma 2.1 since $G^{\prime}(\psi)$ is by Proposition 2.3.

Lastly, since $A_{\beta, \phi^{\perp}}^{\prime}=A_{\beta, \beta^{\perp} \cap R}^{\prime}+A_{\beta, G^{\prime}(\psi)}^{\prime}=0$ we see that $\underline{\beta} \subset \underline{\operatorname{ker}} A_{\phi}^{\prime}$ so that $\phi$ is $\partial^{\prime}$-reducible.

B. As an application of the Reduction Theorem we show how to construct all harmonic maps of $S^{2}$ into $G_{k, n}, k=3,4,5$, from harmonic maps of $S^{2}$ into $C P^{n-1}$ and $G_{2, n}$ which were in turn constructed in $\S 3$.

Theorem 4.2. Let $\phi: S^{2} \rightarrow G_{k, n}$ be a harmonic map, $k=3,4,5$. Then there is a sequence of harmonic maps $\phi_{0}, \cdots, \phi_{N}: S^{2} \rightarrow G_{k, n}$ such that

(i) $\phi_{0}$ is either holomorphic or there is a harmonic map $\psi: S^{2} \rightarrow G_{t, n}$, $1 \leqslant t<k$, and a holomorphic subbundle $\underline{\alpha}$ of $\left(\underline{\psi}+G^{\prime \prime}(\psi)\right)^{\perp}$ such that $\underline{\phi}_{0}=\underline{\psi}$ $\oplus \underline{\alpha}$.

(ii) $\phi_{N}=\phi$.

(iii) For each $i, 0 \leqslant i<N$, there is a holomorphic subbundle $\underline{\alpha}_{i}$ of $\underline{\phi}_{i}$ such that $\underline{\phi}_{i+1}$ is obtained from $\underline{\phi}_{i}$ by forward replacement of $\underline{\alpha}_{i}$.

Remark. Thus to construct any harmonic map $S^{2} \rightarrow G_{k, n}, k=3,4,5$, from the 'holomorphic data' of $\$ 3$ involves the choice of a sequence of holomorphic subbundles (not necessarily of rank one). For simplicity we shall prove only the case $k=3$. The cases $k=4,5$ are similar but longer. 
Again the proof will proceed in several steps. As usual we reverse the orientation of $S^{2}$ for the duration of the proof so as to be able to work with holomorphic second fundamental forms.

By the reduction theorem, it suffices to produce from a given harmonic map $S^{2} \rightarrow G_{3, n}$ a $\partial^{\prime}$-reducible harmonic map. For this we argue as in $\$ 3$ showing how forward replacement of a suitable holomorphic subbundle increases isotropy order.

Proposition 4.3. Let $\phi: S^{2} \rightarrow G_{3, n}$ be harmonic of isotropy order $\geqslant r(r \geqslant 1)$. Suppose that $\phi, G^{\prime}(\phi), \cdots, G^{(r)}(\phi)$ are all $\partial^{\prime}$-irreducible. Then:

(i) The second fundamental form $A_{G^{(r)}(\phi), \phi}^{\prime}$ is holomorphic.

(ii) Let $\underline{\gamma}$ denote $\underline{\operatorname{Im}} A A_{G^{(r)}(\phi), \phi}^{\prime}$; then $\operatorname{rank} \underline{\gamma} \leqslant 2$.

(iii) If rank $\underline{\gamma} \leqslant 1$, then there is a holomorphic subbundle $\underline{\delta}$ of $\phi$ contained in $\operatorname{ker} A_{\phi^{\perp}}^{\prime} \circ A_{\phi}^{\prime}$ forward replacement of which gives a harmonic map $\tilde{\phi}$ of isotropy order $\geqslant r+1$.

(iv) If rank $\gamma=2$, there is a holomorphic subbundle $\underline{\delta}$ of $\phi$ contained in $\operatorname{ker} A_{\phi^{\perp}}^{\prime} \circ A_{\phi}^{\prime}$ forward replacement of which yields a harmonic map $\tilde{\phi}$ of isotropy $\overline{\text { order }} \geqslant r$ such that $\operatorname{rank} A_{G^{(r)}(\tilde{\phi}), \tilde{\phi}}^{\prime} \leqslant 1$.

Corollary 4.4. Let $\phi$ satisfy the hypotheses of Proposition 4.3. Then one or two forward replacements yields a harmonic $\tilde{\phi}$ such that either

(i) $\tilde{\phi}$ has isotropy order $\geqslant r+1$, or

(ii) some $G^{(i)}(\tilde{\phi})$ is $\partial^{\prime}$-reducible, $0 \leqslant i \leqslant r$.

Proof. Arguing exactly as in Proposition 3.4 we have a diagram

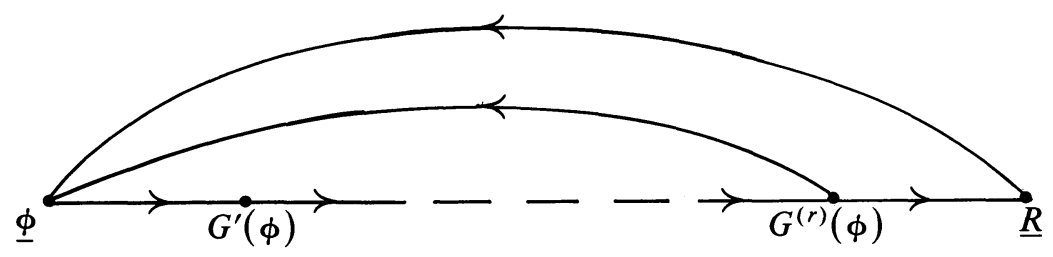

where $\underline{R}=\left(\phi+\cdots+G^{(r)}(\phi)\right)^{\perp}$ and the inner circuit $\left\{\underline{\phi}, G^{\prime}(\phi), \cdots\right.$, $\left.G^{(r)}(\phi), \phi\right\}$ is holomorphic with all vertices of rank 3 . By the irreducibility hypothesis each $A_{G^{(i)}(\phi), G^{(i+1)}(\phi)}^{\prime}$ is an isomorphism for $0 \leqslant i \leqslant r-1$ so that $\underline{\gamma}=\underline{\operatorname{Im}} A_{G^{(r)}(\phi), \phi}^{\prime}$ has rank $\leqslant 2$. This establishes (i) and (ii).

Now suppose rank $\underline{\gamma} \leqslant 1$. Firstly, if $\underline{\gamma}=\underline{0}, G^{(r+1)}(\phi) \perp \underline{\phi}$ so that $\phi$ has isotropy order $\geqslant r+1$ already and we take $\underline{\delta}=\underline{0}$.

So assume that rank $\underline{\gamma}=1$. From Proposition 1.2 we have that $A_{\gamma, G^{\prime}(\phi)}^{\prime}$ is 
holomorphic, so define $\underline{\gamma}_{1}=\underline{\operatorname{Im}} A_{\gamma, G^{\prime}(\phi)}^{\prime}$ and define $\underline{\gamma}_{i}, 1 \leqslant i \leqslant r$, by

$$
\underline{\gamma}_{i}=\underline{\operatorname{Im}} A_{\gamma_{i-1}, G^{(i)}(\phi)}^{\prime} .
$$

Thus each $\underline{\gamma}_{i}$ is a holomorphic rank one subbundle of $G^{(i)}(\phi)$ and each $A_{\gamma_{i}, \gamma_{i+1}}^{\prime}$, $0 \leqslant i \leqslant r-1$, is an isomorphism by the irreducibility hypothesis. Let $\underline{\delta}_{i}=$ $G^{(i)}(\phi) \cap \underline{\gamma}_{i}{ }^{\perp}$. Arguing as in Proposition 3.4 we see that $\left\{\gamma, \gamma_{1}, \cdots, \gamma_{r}, \gamma\right\}$ is a holomorphic circuit so that $A_{\gamma_{r}, \gamma}^{\prime}$ vanishes, whence $\underline{\gamma}_{r} \subset \operatorname{ker} A_{G^{r}(\phi), \phi}^{\prime}$. Thus we have a diagram

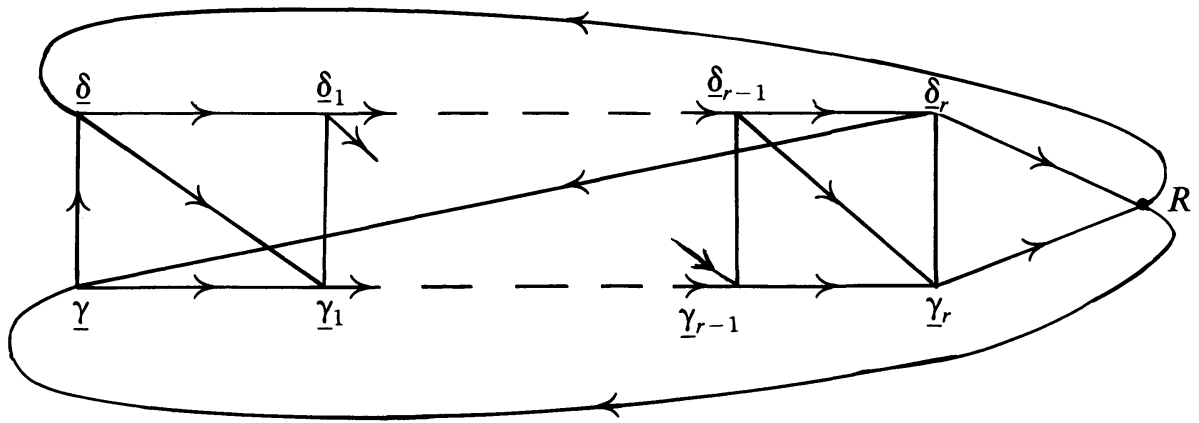

where the horizontal edges are all isomorphisms. By inspection and Proposition 1.5 we see that all the horizontal edges $A_{\gamma_{r}, R}^{\prime}$ and $A_{R, \delta}^{\prime}$ are holomorphic, so we put $\underline{\gamma}_{r+1}=\underline{\operatorname{Im}} A A_{\gamma_{r}, R}^{\prime}$ and $\underline{\delta}_{r+1}=\underline{\gamma}_{r+1}^{\perp} \cap \underline{R}$. Note that $\underline{\gamma}_{r+1}$ is nonzero and $A_{\gamma_{r}, \gamma_{r+1}}^{\prime}$ is an isomorphism since $G^{(r)}(\phi)$ is $\partial^{\prime}$-irreducible. We now have a diagram:

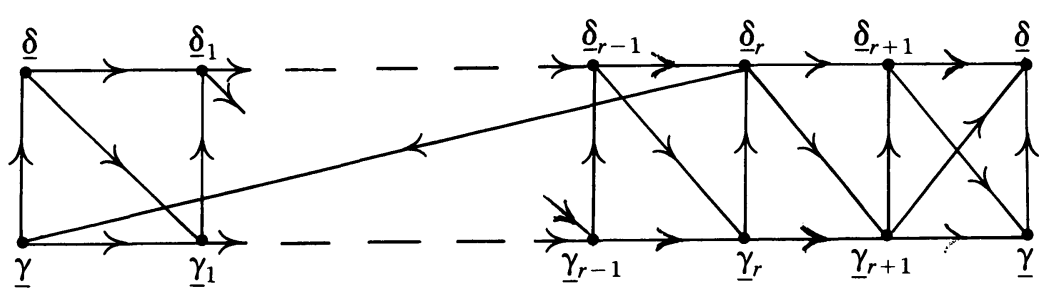

Just as in Proposition 3.4 we have a holomorphic circuit $\left\{\gamma, \gamma_{1}, \cdots, \gamma_{r+1}, \delta\right.$, $\left.\delta_{1}, \cdots, \delta_{r}, \gamma\right\}$ all edges of which are isomorphisms except $A_{\gamma_{r+1}, \delta}^{\prime}$ and $A_{\delta_{r}, \gamma}^{\prime}$ which last is a surjection. Thus either $A_{\gamma_{r+1}, \delta}^{\prime}$ vanishes or its image is contained in the kernel of the composition $\left\{\delta, \delta_{1}, \cdots, \delta_{r}, \gamma\right\}$.

In the first case forward replacement of $\underline{\gamma}$ (clearly in the kernel of $A_{\phi^{\perp}}^{\prime} \circ A_{\phi}^{\prime}$ ) yields a harmonic map of isotropy order $\geqslant r+1$ (cf. Proposition 3.4). Otherwise put $\underline{\eta}=\underline{\operatorname{Im}} A_{\gamma_{r+1}, \delta}^{\prime}$, a holomorphic subbundle of $\underline{\delta}$. Denote by $\underline{\eta}_{i}$ its 
images under the $A_{\delta_{i-1}, \delta_{i}}^{\prime}$ to get a diagram where $\underline{\delta}_{i}^{\prime}=\underline{\eta}_{i}^{\perp} \cap \underline{\delta}_{i}$.

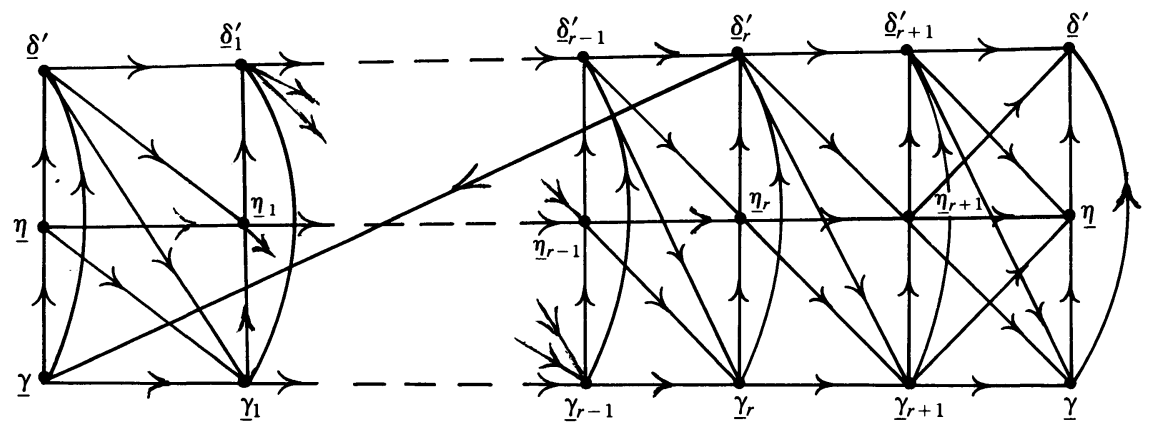

Again inspection shows that the 'zig-zag' circuit $\left\{\gamma, \cdots, \gamma_{r+1}, \eta, \cdots, \eta_{r+1}\right.$, $\left.\delta^{\prime}, \cdots, \delta_{r}^{\prime}, \gamma\right\}$ is holomorphic and, in this circuit, all edges are isomorphisms except $A_{\eta_{r+1}, \delta^{\prime}}^{\prime}$, and all vertices are of rank 1 . Thus $A_{\eta_{r+1}, \delta}^{\prime}$, vanishes.

Now $\operatorname{ker} A_{G^{\prime}(\phi), \phi}^{\prime} \supset \underline{\eta}_{1}+\underline{\gamma}_{1}$ (with equality if $r=1$ ) so that $\underline{\eta}+\underline{\gamma}$ is contained in $\operatorname{ker} A_{\phi^{+}}^{\prime} \circ A_{\phi}^{\prime}$ and so is a candidate for forward replacement. So let $\tilde{\phi}$ be obtained from $\phi$ by forward replacement of $\underline{\eta}+\underline{\gamma}$. Then we have

$$
\begin{aligned}
& \tilde{\Phi}=\underline{\delta}^{\prime}+\underline{\eta}_{1}+\underline{\gamma}_{1}, \\
& G^{(i)}(\tilde{\phi})=\underline{\delta}_{i}^{\prime}+\underline{\eta}_{i+1}+\underline{\gamma}_{i+1} \quad \text { for } 1 \leqslant i \leqslant r \text { and, in particular, } \\
& G^{(r)}(\tilde{\phi})=\underline{\delta}_{r}^{\prime}+\underline{\eta}_{r+1}+\underline{\gamma}_{r+1} .
\end{aligned}
$$

Then since $A_{\eta_{r+1}, \delta}$ vanishes, $G^{(r+1)}(\tilde{\phi}) \subset \underline{\delta}_{r+1}^{\prime}+\underline{\eta}+\underline{\gamma} \perp \tilde{\phi}$ and so $\tilde{\phi}$ has isotropy order $\geqslant r+1$. This concludes the proof of (iii).

For part (iv): Define $\underline{\gamma}_{i}$ and $\underline{\delta}_{i}$ as before so that $\underline{\gamma}_{i}=\underline{\operatorname{Im}} A_{\gamma_{i-1}, G^{(i)}(\phi)}^{\prime}$ and $\underline{\gamma}_{i}^{\perp} \cap G^{i}(\phi)=\underline{\delta}_{i}$ and we get a diagram

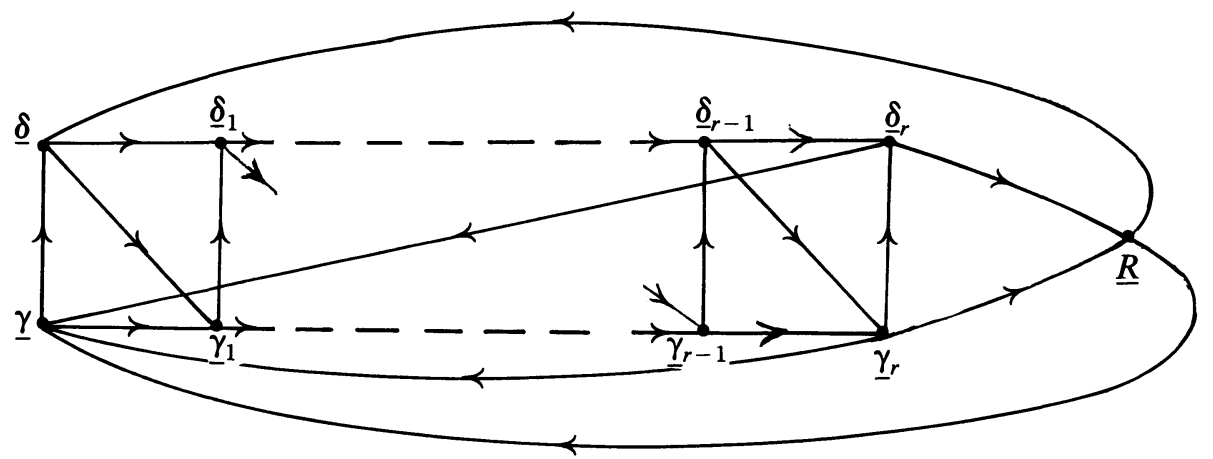

where the circuit $\left\{\gamma, \cdots, \gamma_{r}, \gamma\right\}$ is holomorphic and so rank $A_{\gamma_{r}, \gamma}^{\prime}=1$ since all other edges are isomorphisms and $A_{\gamma_{r}, \gamma}^{\prime}$ cannot vanish without contradicting 
the rank assumption on $A_{G^{(r)}(\phi), \phi}^{\prime}$. So put $\underline{\alpha}=\underline{\operatorname{Im}} A_{\gamma_{r}, \gamma}^{\prime}$ which is holomorphic in $\underline{\gamma}$ and define $\underline{\alpha}_{1}=\underline{\operatorname{Im}} A_{\alpha, \gamma_{1}}^{\prime}, \underline{\alpha}_{i+1}=\underline{\operatorname{Im}} A_{\alpha_{i}, \gamma_{i}}^{\prime}$ for $1 \leqslant i<r, \underline{\beta}_{i}=\underline{\alpha}_{i}^{\perp} \cap \underline{\gamma}_{i}$. Then we have a diagram:

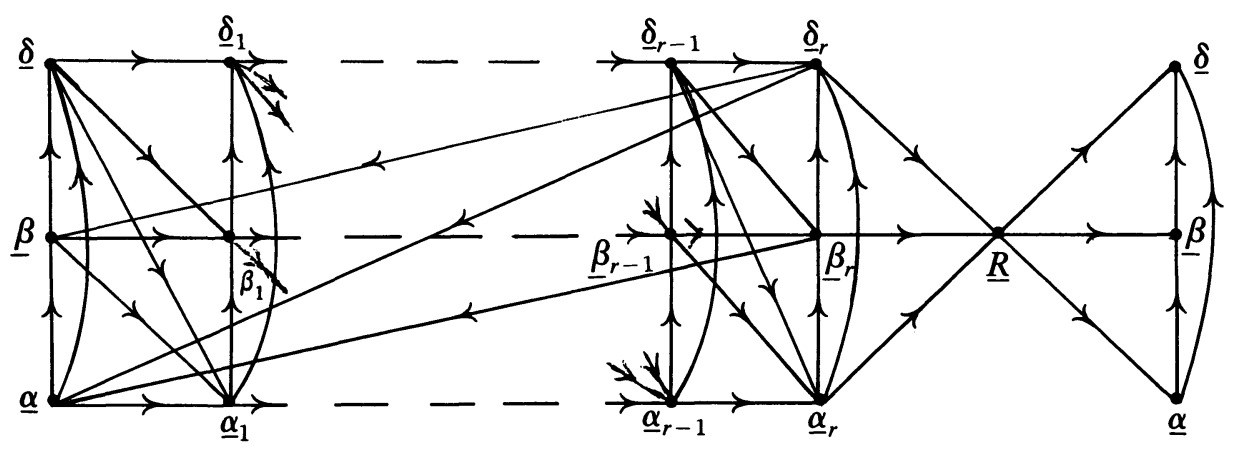

Here we have noted that the circuit $\left\{\alpha, \alpha_{1}, \cdots, \alpha_{r}, \alpha\right\}$ is holomorphic and so since all vertices are of rank one and all horizontal edges are isomorphisms we have $A_{\alpha_{r}, \alpha}^{\prime}=0$. Now from Proposition 1.5 and inspection we see that all $A_{\delta_{i}, \delta_{i+1}}^{\prime}, A_{\beta_{i}, \beta_{i+1}}^{\prime}$ and $A_{\boldsymbol{\alpha}_{i}, \boldsymbol{\alpha}_{i+1}}^{\prime}$ are holomorphic and indeed are isomorphisms by the irreducibility hypothesis. Further $A_{\delta_{r}, \beta}^{\prime}$ and $A_{\beta_{r}, \alpha}^{\prime}$ are holomorphic and are isomorphisms by the assumption on rank $\underline{\gamma}$.

Lastly $A_{\alpha_{r}, R}^{\prime}$ and $A_{R, \delta}^{\prime}$ are holomorphic so that we have a holomorphic circuit $\left\{\alpha, \cdots, \alpha_{r}, R, \delta, \cdots, \delta_{r}, \beta, \cdots, \beta_{r}, \alpha\right\}$ and we conclude that $\operatorname{Im} A_{\alpha_{r}, R}^{\prime}$ $\subset \operatorname{ker} A_{R, \delta}^{\prime}$.

Now it is easy to see that $\underline{\alpha} \subset \operatorname{ker} A_{\phi^{\perp}}^{\prime} \circ A_{\phi}^{\prime}$, so let $\tilde{\phi}$ be obtained from $\phi$ by forward replacement of $\alpha$. Then $\underline{\phi}=\underline{\delta}+\underline{\beta}+\underline{\alpha}_{1}$ and $G^{(r)}(\tilde{\phi})=\underline{\delta}_{r}+\underline{\beta}_{r}$ $+\underline{\operatorname{Im}} A_{\alpha_{r}, R}^{\prime}$. Thus since $A_{R, \delta}^{\prime}\left(\underline{\operatorname{Im}} A_{\alpha_{r}, R}^{\prime}\right)=0$ we see that

$$
\underline{\operatorname{Im}} A_{G^{(r)}(\tilde{\phi})}^{\prime} \subset \underline{\alpha}+\underline{\beta}+\underline{R},
$$

whence $\underline{\operatorname{Im}} A_{G^{(r)}(\tilde{\phi}), \tilde{\phi}}^{\prime}=\underline{\beta}$ and therefore has rank one. This completes the proof of Proposition 4.3.

Proof of Theorem 4.2. Given an arbitrary harmonic map $\phi: S^{2} \rightarrow G_{3, n}$, by Corollary 4.4 and Lemma 3.2 we may forward replace holomorphic subbundles until we obtain a map $\tilde{\phi}: S^{2} \rightarrow G_{3, n}$ which has a $\partial^{\prime}$-reducible $\partial^{\prime}$-Gauss bundle $G^{(i)}(\tilde{\phi})$. It remains to show that $\partial^{\prime}$-Gauss bundles may be obtained by forward replacement but the arguments of Lemma 3.5 carry over word for word to show that $G^{\prime}(\tilde{\phi})$ may be obtained from $\tilde{\phi}$ by at most two forward replacements. Thus we arrive at a $\partial^{\prime}$-reducible map $\tilde{\phi}$ to which the Reduction 
Theorem 4.1 may be applied. It remains only to observe that all replacements used are invertible and to restore the original orientation on $S^{2}$ to complete the proof.

\section{Surfaces of higher genus and other extensions}

A. Degree of a map $\phi: M_{p}^{2} \rightarrow G_{k, n}$. Let $M_{p}^{2}$ denote a compact Riemann surface of genus $p \in\{0,1,2, \cdots\}$ and let $\phi: M_{p}^{2} \rightarrow G_{k, n}$ be a smooth map.

Definition. The degree of $\phi$, denoted $\operatorname{deg} \phi$ is the degree of the induced map $\phi^{*}: H^{2}\left(G_{k, n}, \mathbf{Z}\right) \cong \mathbf{Z} \rightarrow H^{2}\left(M_{p}^{2}, \mathbf{Z}\right) \cong \mathbf{Z}$ on second cohomology. Note that a holomorphic map has nonnegative degree (see, for instance, [13]).

Now let $E \rightarrow M_{p}^{2}$ be a complex vector bundle over $M_{p}^{2}$. We denote by $c_{1}(E)$ the first Chern class of $E$ evaluated on the canonical generator of $H_{2}\left(M_{p}^{2}, \mathbf{Z}\right)$.

Note the minus sign in the following

Lemma 5.1. Let $\phi: M_{p}^{2} \rightarrow G_{k, n}$ be smooth.

(a) $\operatorname{deg} \phi=-c_{1}(\underline{\phi})=-c_{1}\left(\wedge^{k} \phi\right)$.

(b) $\operatorname{deg} \phi^{\perp}=-\operatorname{deg} \phi$.

Proof. Let $T \rightarrow G_{k, n}$ be the tautological $k$-plane bundle over $G_{k, n}$. The first Chern class of $T$ evaluated on the canonical generator of $H^{2}\left(G_{k, n}, \mathbf{Z}\right)$ is -1 . Thus, by the functoriality of Chern classes we have

$$
c_{1}(\underline{\phi})=c_{1}\left(\phi^{-1} T\right)=-\operatorname{deg} \phi .
$$

The other assertions follow from well-known properties of Chern classes (see, for example, Hirzeburch [22]). q.e.d.

To obtain vanishing second fundamental forms for surfaces of higher genus we need

Lemma 5.2. Let $E, F$ be holomorphic vector bundles over $M_{p}$ of the same rank $r$ and let $\mathscr{A}=d z^{k} \otimes A$ be a holomorphic section of $\otimes{ }^{k} T_{(1,0)}^{*} M_{p} \otimes L(E, F)$. Then if $A$ is an isomorphism on any fibre,

$$
c_{1}(F) \geqslant c_{1}(E)+r k(2-2 p)
$$

In particular, if $E$ and $F$ have rank one and $c_{1}(F)<c_{1}(E)+k(2-2 p)$, then $A$ is identically zero on each fibre.

Proof. $\wedge^{r} \mathscr{A}$ is a holomorphic section of the line bundle $L=\otimes^{r k} T_{(1,0)}^{*} M_{p}$ $\otimes L\left(\wedge^{r} E, \wedge^{r} F\right)$. Now

$$
c_{1}(L)=-r k(2-2 p)-c_{1}(E)+c_{1}(F)
$$

and if this is negative, $\wedge^{r} \mathscr{A}$ vanishes identically so that $A$ cannot be an isomorphism on any fibre. 
B. Inclusive harmonic maps $M_{p} \rightarrow G_{2, n}$. In this section we consider harmonic maps $\phi: M^{2} \rightarrow G_{2, n}$ such that $\phi^{\perp}$ is strongly conformal. Such maps, if non- \pm holomorphic, are precisely the 'inclusive maps' in the sense of Eells-Salamon [14] with respect to the quaternionic Kähler structure of $G_{2, n}$ (see below). For surfaces of low genus such maps are often very easy to describe.

Proposition 5.3. Let $\phi: S^{2} \rightarrow G_{2, n}$ be harmonic and suppose $\phi^{\perp}: S^{2} \rightarrow$ $G_{n-2, n}$ is strongly conformal. Then $\phi$ is \pm holomorphic, a Frenet pair or a mixed pair. In particular either $\phi$ or $\phi^{\perp}$ is strongly isotropic.

Proof. If $\phi^{\perp}$ is \pm holomorphic there is nothing to prove so we suppose that this is not the case. Then $G^{\prime}\left(\phi^{\perp}\right)$ and $G^{\prime \prime}\left(\phi^{\perp}\right)$ are mutually orthogonal and must have rank one. Denote these bundles by $\underline{\alpha}, \underline{\beta}$ respectively. Then we have a diagram

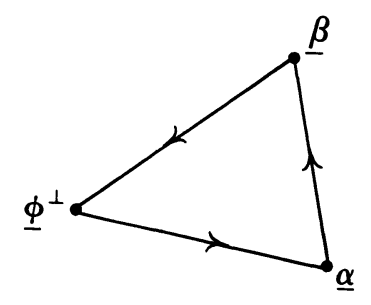

with $A_{\phi^{\perp}, \alpha}^{\prime}$ and $A_{\beta, \phi^{\perp}}^{\prime}$ nonzero. If $A_{\alpha, \beta}^{\prime}$ vanishes, then $\phi$ is the mixed pair $\underline{\alpha} \oplus \underline{\beta}$. Otherwise, we have a holomorphic circuit with all edges of rank one so that ${ }^{\prime} \phi_{\phi^{\perp}, \alpha}^{\circ} A_{\beta, \phi^{\perp}}^{\prime}=0$, i.e., $\operatorname{Im} A_{\beta, \phi^{\perp}}^{\prime} \subset \underline{\operatorname{ker}} A_{\phi^{\perp}, \alpha}^{\prime}$. So set $\underline{\gamma}_{1}=\underline{\operatorname{ker}} A_{\phi^{\perp}, \alpha}^{\prime}, \underline{\delta}_{1}=$ $\underline{\gamma}_{1}^{\perp} \cap \underline{\phi}^{\perp}$ to obtain a diagram

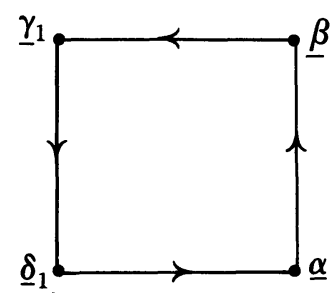

where rank $\delta_{1}=1$ and all edges except possibly $A_{\gamma_{1}, \delta_{1}}^{\prime}$ are nonzero. If $A_{\gamma_{1}, \delta_{1}}^{\prime}=0$, then $\underline{\delta}_{1}$ is holomorphic and $\phi=\underline{\alpha} \oplus \underline{\beta}$ is an associated Frenet pair. Otherwise, again, we have a holomorphic circuit and $\underline{\operatorname{Im}} A_{\beta, \gamma_{1}}^{\prime} \subset \underline{\operatorname{ker}} A_{\gamma_{1}, \delta_{1}}^{\prime}$. In this case set 
$\underline{\gamma}_{2}=\underline{\operatorname{ker}} A_{\gamma_{1}, \delta_{1}}^{\prime}, \underline{\delta}_{2}=\underline{\gamma}_{2}^{\perp} \cap \underline{\gamma}_{1}$ and we have a diagram

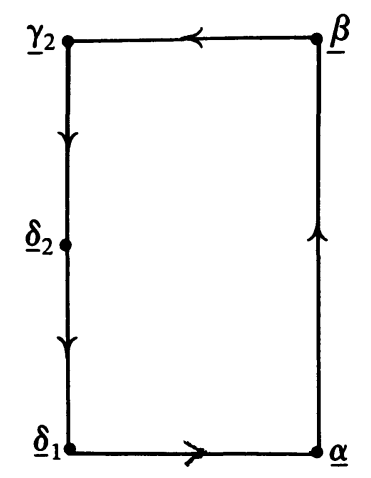

with all vertices of rank one except $\gamma_{2}$ and all edges nonzero except possibly $A_{\gamma_{2}, \delta_{2}}^{\prime}$. We repeat this procedure reducing the dimension of $\gamma_{i}$ each time so eventually for some $i, A_{\gamma_{i}, \delta_{i}}^{\prime}$ vanishes and we have a diagram

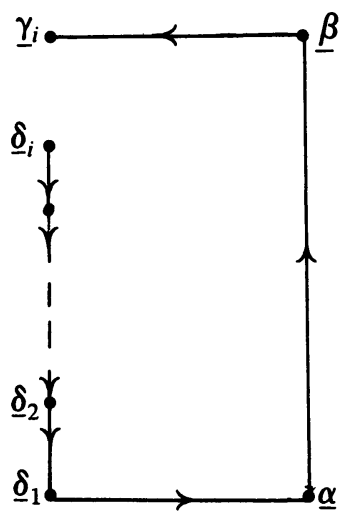

so that $\underline{\delta}_{i}$ is holomorphic and $\underline{\phi}=G^{(i)}\left(\delta_{i}\right) \oplus G^{(i+1)}\left(\delta_{i}\right)$.

Thus $\phi$ is a Frenet pair and the proposition is proved.

Adding a hypothesis on degree we can prove a similar result for maps $T^{2} \rightarrow G_{2, n}$.

Theorem 5.4. Let $\phi: T^{2} \rightarrow G_{2, n}$ be a harmonic map of odd degree. Suppose that $\phi^{\perp}$ is strongly conformal. Then $\phi$ is \pm holomorphic, a Frenet pair or a mixed pair. 
Proof. Assuming that $\phi$ is not \pm holomorphic and not a mixed pair we see that just as in Proposition 5.3 we have a diagram

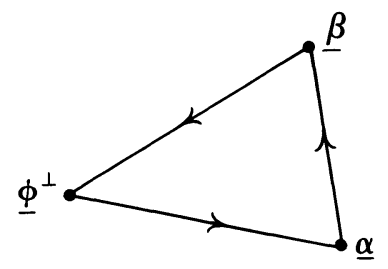

with all edges nonzero. In particular $d z \otimes A_{\alpha, \beta}^{\prime}$ is nonzero so that $c_{1}(\underline{\beta}) \geqslant$ $c_{1}(\underline{\alpha})+0$ by Lemma 5.2. Now if $A_{\phi^{\perp}, \alpha}^{\prime} A_{\beta, \phi^{\perp}}^{\prime} \neq 0, d z^{2} \otimes A_{\phi^{\perp}, \alpha}^{\prime}{ }^{\circ} A_{\beta, \phi^{\perp}}^{\prime}$ is nonzero so that, again by Proposition 5.3, $c_{1}(\underline{\alpha}) \geqslant c_{1}(\underline{\beta})+0$, whence $c_{1}(\underline{\alpha})=$ $c_{1}(\underline{\beta})$ and $\operatorname{deg} \phi=-\left(c_{1}(\underline{\alpha})+c_{1}(\underline{\beta})\right)$ is even. Thus if $\phi$ has odd degree $\operatorname{Im} A_{\beta, \phi^{\perp}}^{\prime}$ $\subset \overline{\operatorname{ker}} A_{\phi^{\perp}, \alpha}^{\prime}$ and setting $\underline{\gamma}_{1}=\underline{\operatorname{ker}} A_{\phi^{\perp}, \alpha}^{\prime}$ and $\underline{\delta}_{1}=\underline{\gamma}_{1}{ }^{\perp} \cap \underline{\phi}^{\perp}$ we get diagram (1) of Proposition 5.3. Again, if $\bar{A}_{\gamma_{1}, \delta_{1}}^{\prime} \circ A_{\beta, \gamma_{1}}^{\prime} \neq 0$ we get $c_{1}(\underline{\alpha}) \geqslant c_{1}(\underline{\beta})$ so we repeat the argument of Proposition 5.3 to conclude that $\phi$ is a Frenet pair.

C. Application to $\mathbf{H} \boldsymbol{P}^{n}$. A quaternionic Käher manifold, $N$, is a $4 n$ dimensional Riemannian manifold whose holonomy group is contained in $\operatorname{Sp}(n) \operatorname{Sp}(1) \subset \mathrm{SO}(4 n)$. Thus $\operatorname{End}(T N)$ has a rank 3 subbundle $Q$, stable under covariant differentiation, locally spanned by sections $I, J, K$ satisfying

(i) $I^{2}=J^{2}=K^{2}=-I d$,

(ii) $I J=K, J K=I, K I=J$,

(iii) $I, J, K$ skew-symmetric.

A subspace $W \subset T_{x} N$ is called quaternionic if $Q_{x} W \subset W$.

Definition. A map $\phi: M^{2} \rightarrow N$ is inclusive if $d \phi\left(T_{x} M\right)$ is contained in a 4 (real) dimensional quaternionic subspace of $T_{\phi(x)} N$ for each $x \in M$. For more information on quaternionic Kähler manifolds and inclusive maps see Salamon [28], [29] and Eells-Salamon [14].

Now $G_{2, n}$ is quaternionic Kähler with the bundle $Q$ being given by right composition with skew Hermitian endomorphisms of $T$, the tautological 2-plane bundle. (Here, of course, we identify $T G_{2, n}$ with $L\left(T, T^{\perp}\right)$.)

Rawnsley has shown

Lemma 5.5. A weakly conformal non- \pm holomorphic map $\phi: M^{2} \rightarrow G_{2, n}$ is inclusive if and only if $\phi^{\perp}: M^{2} \rightarrow G_{2, n}$ is strongly conformal.

Thus Proposition 5.3 and Theorem 5.4 apply and we have

Theorem 5.6. Let $\phi: S^{2} \rightarrow G_{2, n}$ be harmonic and inclusive. Then $\phi$ is \pm holomorphic, a Frenet pair, or a mixed pair.

The same conclusion holds if $\phi: T^{2} \rightarrow G_{2, n}$ is inclusive conformal and harmonic of odd degree. 
Now let $\mathbf{H} P^{n-1}$ denote quaternionic projective $(n-1)$ space which we regard as the subset of $G_{2,2 n}$ consisting of complex 2-planes $W \in G_{2,2 n}$ closed under the action of $J: \mathbf{C}^{2 n} \rightarrow \mathbf{C}^{2 n}$ given by

$$
J(x, y)=(-\bar{y}, \bar{x}), \quad(x, y) \in \mathbf{C}^{n} \times \mathbf{C}^{n}=\mathbf{C}^{2 n} .
$$

Under the identification $\mathbf{C}^{2 n} \simeq \mathbf{H}^{n}$ given by $(x, y) \mapsto(x+y j), J$ is just left multiplication by $j$.

Proposition 5.7. A map $\phi: M^{2} \rightarrow \mathbf{H} P^{n-1}$ is inclusive if and only if $\phi$ is d'-reducible.

Proof. We have (i) $J A_{\phi^{\perp}}^{\prime \prime}=A_{\phi^{\perp}}^{\prime} J$, whence $J G^{\prime \prime}\left(\phi^{\perp}\right)=G^{\prime}\left(\phi^{\perp}\right)$; (ii) $\langle x, J x\rangle$ $=0$ for all $x \in \mathbf{C}^{2 n}$. Thus from (i) we see that $\phi$ is \pm holomorphic if and only if $\phi$ is constant in which case the proposition is trivially true.

Now $\phi \cap G^{\prime \prime}\left(\phi^{\perp}\right)^{\perp}=\operatorname{ker} A_{\phi}^{\prime}$ so that if $\phi$ is nonconstant and $\partial^{\prime}$-reducible rank $G^{\prime \prime}\left(\phi^{\perp}\right)=1$, and from (i) and (ii) we have $G^{\prime \prime}\left(\phi^{\perp}\right) \perp G^{\prime}\left(\phi^{\perp}\right)$, whence $\phi^{\perp}$ is strongly conformal, hence $\phi$ is inclusive. Conversely if $\phi^{\perp}$ is strongly conformal and nonconstant, again rank $G^{\prime \prime}\left(\phi^{\perp}\right)=1$ whence $\phi$ is $\partial^{\prime}$-reducible. q.e.d.

Thus we recover a theorem of Aithal [1] in a slightly more explicit form:

Theorem 5.8 (cf. [1]). Let $\phi: S^{2} \rightarrow \mathbf{H} P^{n-1}$ be harmonic, $\partial^{\prime}$-reducible, and nonconstant. Then $\phi$ is a Frenet pair or a mixed pair. In particular, $\phi$ or $\phi^{\perp}$ is strongly isotropic.

Remark. Theorem 5.4 has no application to maps $\phi: T^{2} \rightarrow \mathbf{H} P^{n-1}$ since $H^{2}\left(\mathbf{H} P^{n-1}, \mathbf{Z}\right)=0$, so $\operatorname{deg} \phi=0$.

D. Maps into $G_{2,4^{*}}$.

Proposition 5.9. Let $\phi: M_{p}^{2} \rightarrow G_{k, 2 k}$ be a harmonic map.

(a) If $\operatorname{deg}(\phi)<k(1-p), \phi$ is $\partial^{\prime}$-reducible and $\phi^{\perp}$ is $\partial^{\prime \prime}$-reducible.

(b) If $\operatorname{deg}(\phi)>k(p-1), \phi$ is $\partial^{\prime \prime}$-reducible and $\phi^{\perp}$ is $\partial^{\prime}$-reducible.

Thus if $p=0$ or $|\operatorname{deg} \phi|>k(p-1)$, either $\phi$ or $\phi^{\perp}$ is $\partial^{\prime}$-reducible.

Proof. If $A_{\phi}^{\prime}$ or, equivalently $A_{\phi^{\perp}}^{\prime \prime}$, is an isomorphism on some fibre, then by Lemma 5.2

$$
c_{1}\left(\underline{\phi}^{\perp}\right) \geqslant c_{1}(\underline{\phi})+k(2-2 p)
$$

or, by Lemma 5.1,

$$
\operatorname{deg}(\phi) \geqslant k(1-p)
$$

Thus (a) is proved. Part (b) is similar. q.e.d.

Specializing to $k=2$ and applying the Reduction Theorem 4.1, we have

Theorem 5.10. Let $\phi: M_{p}^{2} \rightarrow G_{2,4}$ be a non- \pm holomorphic harmonic map. Then if either (i) $p=0$ or, (ii) $p \geqslant 1$ and $|\operatorname{deg} \phi|>k(p-1)$, then there is a harmonic map $\psi: M_{p} \rightarrow \mathbf{C P} P^{3}$ and an antiholomorphic line subbundle $\underline{\beta}$ of $\left(\underline{\psi}+G^{\prime}(\psi)\right)^{\perp}$ such that either $\phi$ or $\phi^{\perp}$ is given by $\underline{\psi} \oplus \underline{\beta}$. 
Remarks. (i) Choice of $\underline{\beta}$ is equivalent to the choice of a holomorphic section of a $\mathbf{C} P^{1}$ bundle.

(ii) If $p=0$, then $M_{p}=S^{2}$ and (5.10) applies to any non- \pm holomorphic $\operatorname{map} S^{2} \rightarrow G_{2,4}$.

Further, in this case we know the structure of $\psi$ (see $\$ 2 D)$ and we obtain the theorem of Ramanathan [25]:

Let $\phi: S^{2} \rightarrow G_{2,4}$ be a non- \pm holomorphic harmonic map. Then either $\phi$ or $\phi^{\perp}$ is the sum $G^{(i)}(h) \oplus \beta$, where $h: S^{2} \rightarrow \mathbf{C} P^{3}$ is a holomorphic map and $\underline{\beta}$ is an antiholomorphic subbundle of $\left(G^{(i)}(h)+G^{(i+1)}(h)\right)^{\perp}, i \in\{0,1,2\}$.

(iii) If $p=1,(5.10)$ applies if $\operatorname{deg} \phi \neq 0$ (cf. [17, Proposition 7.6]).

(iv) For $p=0$ and $|\operatorname{deg} \phi| \leqslant 1$ we see from Proposition 5.9 with $k=2$ that $\phi$ and $\phi^{\perp}$ are both $\partial^{\prime}$ and $\partial^{\prime \prime}$-reducible. In fact we can say more in this case:

Theorem 5.11. Let $\phi: S^{2} \rightarrow G_{2,4}$ be harmonic with $|\operatorname{deg} \phi| \leqslant 1$. Then either $\phi$ or $\phi^{\perp}$ is \pm holomorphic, a Frenet pair or a mixed pair.

To prove Theorem 5.11 it suffices to show that either $\phi$ or $\phi^{\perp}$ is strongly conformal. This in turn follows from Proposition 5.9 (see above Remark) and the following lemma of possibly independent interest.

Lemma 5.12. Let $\phi: M_{p} \rightarrow G_{2,4}$ be a $\partial^{\prime}$-reducible weakly conformal harmonic map of degree $>2 p-2$. Then either $\phi$ or $\phi^{\perp}$ is strongly conformal.

Proof. If $\phi$ is \pm holomorphic there is nothing to prove. Otherwise set $\underline{\beta}$ $=\underline{\operatorname{ker}} A_{\phi}^{\prime}, \underline{\alpha}=\underline{\beta}^{\perp} \cap \underline{\phi}, \underline{\gamma}=\underline{\operatorname{Im}} A_{\phi}^{\prime}$ and $\underline{\delta}=\underline{\gamma}^{\perp} \cap \underline{\phi}^{\perp}$.

Now all these bundles have rank one and by definition of $\beta, \gamma$, the only nonzero component of $A_{\phi}^{\prime}$ is $A_{\alpha, \gamma}^{\prime}$. Further, by the conformality hypothesis

$$
\operatorname{trace}\left(A_{\phi^{\perp}}^{\prime} \circ A_{\phi}^{\prime}\right)=\operatorname{trace} A_{\gamma, \alpha}^{\prime} \circ A_{\alpha, \gamma}^{\prime}=0,
$$

whence $A_{\gamma, \alpha}^{\prime}=0$ and we have a diagram:

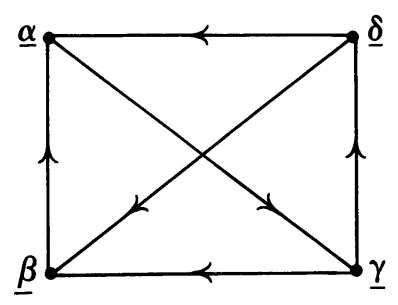

By Proposition 1.5 we see that $A_{\gamma, \beta}^{\prime}$ and $A_{\delta, \alpha}^{\prime}$ are holomorphic so that if neither vanish identically we have, from Lemma 5.2,

$$
c_{1}(\underline{\alpha}) \geqslant c_{1}(\underline{\delta})+2-2 p, \quad c_{1}(\underline{\beta}) \geqslant c_{1}(\underline{\gamma})+2-2 p
$$

and adding and using $c_{1}(\underline{\phi})=c_{1}(\underline{\alpha})+c_{1}(\underline{\beta})$ we have

$$
c_{1}(\underline{\phi}) \geqslant c_{1}\left(\underline{\phi}^{\perp}\right)+4-4 p
$$


or equivalently,

$$
\operatorname{deg}(\phi) \leqslant 2 p-2 \text {. }
$$

Thus if $\operatorname{deg}(\phi)>2 p-2$, either $A_{\gamma, \beta}^{\prime}$ or $A_{\delta, \alpha}^{\prime}$ vanishes. If $A_{\gamma, \beta}^{\prime}=0$ then $G^{\prime}(\phi) \subset \underline{\gamma}, G^{\prime \prime}(\phi) \subset \underline{\delta}$ and $\phi$ is strongly conformal. Similarly, if $A_{\delta, \alpha}^{\prime}=0, \phi^{\perp}$ is strongly conformal.

Remark. Note that the case $p=0,|\operatorname{deg} \phi|<1$ is the only one where the hypotheses Proposition 5.9(a) and Lemma 5.12 hold simultaneously.

E. Nilpotency and degree. For a harmonic map $\phi: S^{2} \rightarrow G_{k, n}$ it is clear that the nilpotency of $A_{\phi^{\perp}}^{\prime} \circ A_{\phi}^{\prime}$ is an important ingredient in our constructions. We now examine the possibility of such nilpotency for maps from surfaces of higher genus.

Proposition 5.13. Let $\phi: M_{p}^{2} \rightarrow G_{k, n}$ be harmonic and $\partial^{\prime}$-irreducible and let $\underline{\gamma}=G^{\prime}(\phi)$. Then if $\operatorname{deg} \gamma-\operatorname{deg} \phi<k(2-2 p), A_{\phi^{\perp}}^{\prime} \circ A_{\phi}^{\prime}$ is not an isomorphism on any fibre.

Proof. Since $\phi$ is $\partial^{\prime}$-irreducible, $\operatorname{rank} \phi=\operatorname{rank} \underline{\gamma}=k$ and $A_{\gamma, \phi}^{\prime}$ is holomorphic. Thus if $A_{\gamma, \phi}^{\prime}$ is an isomorphism on any fibre, we have, from Lemma 5.2,

$$
c_{1}(\underline{\phi}) \geqslant c_{1}\left(G^{\prime}(\phi)\right)+k(2-2 p) .
$$

Thus if $\operatorname{deg} \gamma-\operatorname{deg} \phi<k(2-2 p), A_{\gamma, \phi}^{\prime}$ and $A_{\phi^{\perp}}^{\prime} \circ A_{\phi}^{\prime}$ are not isomorphisms on any fibre.

Corollary 5.14. Let $\phi: M_{p}^{2} \rightarrow G_{2, n}$ be $\partial^{\prime}$-irreducible, weakly conformal and harmonic with $\operatorname{deg} \gamma-\operatorname{deg} \phi<2(2-2 p)$. Then $A_{\phi^{\perp}}^{\prime} \circ A_{\phi}^{\prime}$ is nilpotent.

Proof. The coefficients of the characteristic polynomial of $A_{\phi^{\perp}}^{\prime} \circ A_{\phi}^{\prime}$ are trace $\left(A_{\phi^{\perp}}^{\prime} \circ A_{\phi}^{\prime}\right)$ which vanishes by weak conformality and $\operatorname{det}\left(A_{\phi^{\perp}}^{\prime} \circ A_{\phi}^{\prime}\right)$ which vanishes by Proposition 5.13, thus $A_{\phi^{\perp}}^{\prime} \circ A_{\phi}^{\prime}$ is nilpotent. q.e.d.

As an application we extend to surfaces of higher genus a theorem of Aithal [2] concerning maps into $G_{2,5}$.

Theorem 5.15. Let $\phi: M_{p} \rightarrow G_{2,5}$ be a harmonic map of degree $<2(2-2 p)$. Suppose that $A_{\phi^{\perp}}^{\prime} \circ A_{\phi}^{\prime}$ is nilpotent. Then either $\phi$ or $G^{\prime}(\phi)$ is $\partial^{\prime}$-reducible. Thus one of the following holds:

(i) $\phi$ is antiholomorphic.

(ii) There is a harmonic map $\psi: M_{p}^{2} \rightarrow \mathbf{C} P^{4}$ and an antiholomorphic subbundle $\beta$ of $\left(\psi+G^{\prime}(\psi)\right)^{\perp}$ and

$$
\underline{\phi}=\underline{\psi} \oplus \underline{\beta} \quad \text { or } \quad \underline{\phi}=G^{\prime \prime}(\underline{\psi} \oplus \underline{\beta}) .
$$

(iii) $\underline{\phi}=G^{\prime \prime}(\psi)$, where $\psi: M_{p}^{2} \rightarrow G_{2,5}$ is antiholomorphic.

From Corollary 5.14 we have

Corollary 5.16. Let $\phi: M_{p} \rightarrow G_{2,5}$ be weakly conformal and harmonic with $\operatorname{deg} \phi<2(2-2 p)$ and $\operatorname{deg} \gamma-\operatorname{deg} \phi<2(2-2 p)$. Then either $\phi$ or $G^{\prime}(\phi)$ is $\partial^{\prime}$-reducible and one of (i)-(iii) in Theorem 5.15 hold. 
Proof of Theorem 5.15. It suffices to show $\partial^{\prime}$-reducibility of $\phi$ or $G^{\prime}(\phi)$, the rest following from the Reduction Theorem.

Suppose then that $\phi$ is $\partial^{\prime}$-irreducible, set $\underline{\gamma}=G^{\prime}(\phi)$ and consider $A_{\gamma, \phi}^{\prime}$. If it is zero, then rank $A_{\gamma}^{\prime} \leqslant 1$ and $\underline{\gamma}$ is $\partial^{\prime}$-reducible; so suppose that rank $A_{\gamma, \phi}^{\prime}=1$. Put $\underline{\beta}_{1}=\operatorname{ker} A_{\gamma, \phi}^{\prime}, \underline{\beta}_{2}=\underline{\beta}_{1}{ }^{\perp} \cap \underline{\gamma}, \underline{\alpha}_{1}=\underline{\operatorname{Im}} A_{\gamma, \phi}^{\prime}, \underline{\alpha}_{2}=\underline{\alpha}_{1}{ }^{\perp} \cap \underline{\phi}, \underline{R}=\left(\underline{\alpha}_{1} \oplus \underline{\alpha}_{2} \oplus\right.$ $\left.\underline{\beta}_{1} \oplus \underline{\beta}_{2}\right)^{\perp}$. By definition the only nonzero component of $A_{\gamma, \phi}^{\prime}$ is $A_{\beta_{2}, \alpha_{1}}^{\prime}$ and from the nilpotency of $A_{\phi^{\perp}}^{\prime} \circ A_{\phi}^{\prime}$ it is easy to see that $A_{\alpha_{1}, \beta_{2}}^{\prime}$ vanishes. Thus we have a diagram

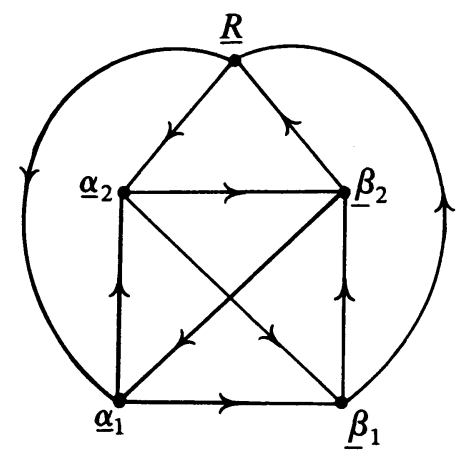

and from Proposition 1.5 we see that the following second fundamental forms are holomorphic: $A_{\alpha_{2}, \beta_{2}}^{\prime}, A_{\alpha_{1}, \beta_{1}}^{\prime}, A_{\beta_{2}, \alpha_{1}}^{\prime}$ and $A_{\beta_{1}, R}^{\prime}$. Further, the first three are nonzero since $\phi$ is $\partial^{\prime}$-irreducible and rank $A_{\gamma, \phi}^{\prime}=1$. If $A_{\beta_{1}, R}^{\prime} \neq 0$ also we have from Lemma 5.2,

$$
\begin{aligned}
& c_{1}\left(\underline{\beta}_{1}\right) \geqslant c_{1}\left(\underline{\alpha}_{1}\right)+(2-2 p), \\
& c_{1}\left(\underline{\beta}_{2}\right) \geqslant c_{1}\left(\underline{\alpha}_{2}\right)+(2-2 p), \\
& c_{1}\left(\underline{\alpha}_{1}\right) \geqslant c_{1}\left(\underline{\beta}_{2}\right)+(2-2 p), \\
& c_{1}(\underline{R}) \geqslant c_{1}\left(\underline{\beta}_{1}\right)+(2-2 p),
\end{aligned}
$$

whence

$$
c_{1}\left(\underline{\alpha}_{1}\right) \geqslant c_{1}\left(\underline{\alpha}_{2}\right)+2(2-p) \text {, }
$$

whence

$$
c_{1}(\underline{R}) \geqslant c_{1}\left(\underline{\alpha}_{1}\right)+2(2-p) \geqslant \frac{1}{2} c_{1}\left(\underline{\alpha}_{1}\right)+(2-2 p)+\frac{1}{2} c_{1}\left(\underline{\alpha}_{2}\right)+2(2-2 p),
$$

so that

$$
c_{1}\left(\underline{\phi}^{\perp}\right)=c_{1}\left(\underline{\beta}_{1}\right)+c_{1}\left(\underline{\beta}_{2}\right)+c_{1}(\underline{R}) \geqslant \frac{3}{2}\left(c_{1}\left(\underline{\alpha}_{1}\right)+c_{1}\left(\underline{\alpha}_{2}\right)\right)+5(2-2 p),
$$


i.e.,

$$
\frac{5}{2} \operatorname{deg} \phi \geqslant 5(2-2 p) .
$$

Thus if $\operatorname{deg} \phi<2(2-2 p)$ we have $A_{\beta_{1}, R}^{\prime}=0$, whence $\underline{\beta}_{1} \subset \underline{\operatorname{ker}} A_{\gamma}^{\prime}$ and $\underline{\gamma}=$ $G^{\prime}(\phi)$ is $\partial^{\prime}$-reducible.

Remark. It is clear from the foregoing that harmonic maps of Riemann surfaces of higher genus into Grassmannians will be considerably harder to study than those from $S^{2}$.

\section{References}

[1] A. R. Aithal, Harmonic maps from $S^{2}$ to $\mathbf{H} P^{2}$, Osaka J. Math., to appear.

[2] Harmonic maps from $S^{2}$ to $G_{2}\left(C^{5}\right)$, J. London Math. Soc., to appear.

[3] D. Burns, Harmonic maps from $\mathbf{C} P^{1}$ to $\mathbf{C} P^{n}$, in Harmonic Maps (Proceedings, New Orleans 1980, 48-56), Lecture Notes in Math. , Vol. 949, Springer, Berlin, 1982.

[4] F. E. Burstall, A twistor description of harmonic maps of a 2-sphere into a Grassmannian, Math. Ann. to appear.

[5] - Twistor fibrations of flag manifolds and harmonic maps of a 2-sphere into a Grassmannian, Differential Geometry, ed. L. A. Cordero, Research Notes in Math., 131, Pitman, London (1985) 7-16.

[6] E. Calabi, Minimal immersions of surfaces in Euclidean spheres, J. Differential Geometry 1 (1967) 111-125.

[7] S.-S. Chern \& J. Wolfson, Minimal surfaces by moving frames, Amer. J. Math. 105 (1983) 59-83.

[8] Harmonic maps of $S^{2}$ into a complex Grassmannian manifold, Proc. Nat. Acad. Sci. U.S.A. 82 (1985) 2217-2219; II, preprint, Berkeley and Tulane University.

[9] A. M. Din \& W. J. Zakrzewski, General classical solutions in the $\mathbf{C} P^{n-1}$ model, Nuclear Phys. B174 (1980) 397-406.

[10] _ Classical solutions in Grassmannian o-models, Lett. Math. Phys. 5 (1981) 553-561.

[11] J. Eells, Gauss maps of surfaces, Perspectives in Math., Anniversary of Oberwolfach, Birkhäuser, Basel, 1984.

[12] J. Eells \& L. Lemaire, A report on harmonic maps, Bull. London Math. Soc. 10 (1978) 1-68.

[13] _ Selected topics in harmonic maps, C.B.M.S. Regional Conf. Ser. in Math., no. 50, Amer. Math. Soc., Providence, RI, 1983.

[14] J. Eells \& S. Salamon, Constructions twistorielles des applications harmoniques, C.R. Acad. Sci. Paris I, 296 (1983) 685-687.

[15] _ Twistorial construction of harmonic maps of surfaces into four-manifolds, Ann. Scuola Norm. Sup. Pisa, to appear.

[16] J. Eells \& J. H. Sampson, Harmonic mappings of Riemannian manifolds, Amer. J. Math. 86 (1964) 109-160.

[17] J. Eells \& J. C. Wood, Harmonic maps from surfaces to complex projective spaces, Advances in Math. 49 (1983) 217-263.

[18] S. Erdem \& J. F. Glazebrook, Harmonic maps of Riemann surfaces to indefinite complex hyperbolic and projective spaces, Proc. London Math. Soc. (3) 47 (1983) 547-562.

[19] S. Erdem \& J. C. Wood, On the construction of harmonic maps into a Grassmannian, J. London Math. Soc. (2) 28 (1983) 161-174.

[20] V. Glaser \& R. Stora, Regular solutions of the $\mathbf{C} P^{n}$ models and further generalizations, preprint, CERN, 1980. 
[21] J. F. Glazebrook, The construction of a class of harmonic maps to quaternionic projective space, J. London Math. Soc. (2) 30 (1984) 151-159.

[22] F. Hirzebruch, Topological methods in algebraic geometry, Grundlehren 131, Springer, Berlin, 1966.

[23] J. L. Koszul \& B. Malgrange, Sur certaines structures fibrées complexes, Arch. Math. 9 (1958) 102-109.

[24] A. Lichnerowicz, Applications harmoniques et variétés kählériennes, Sympos. Math. III, Bologna, 1970, 341-402.

[25] J. Ramanathan, Harmonic maps from $S^{2}$ to $G_{2,4}$, J. Differential Geometry 19 (1984) 207-219.

[26] J. Rawnsley, f-structures, f-twistor spaces and harmonic maps, Geometry Seminar Luigi Bianchi. II, Lecture Notes in Math., Vol. 1164, Springer, Berlin.

[27] E. Ruh \& J. Vilms, The tension field of the Gauss map, Trans. Amer. Math. Soc. 149 (1970) 569-573.

[28] S. Salamon, Quaternionic Kähler manifolds, Invent. Math., 67 (1982) 143-171.

[29] Harmonic and holomorphic maps, Geometry Seminar Luigi Bianchi. II, Lecture Notes in Math., Vol. 1164, Springer, Berlin.

[30] _ Minimal surfaces and symmetric spaces Differential Geometry, ed. L. A. Cordero, Research Notes in Math., 131, Pitman, London (1985) 103-114.

[31] K. K. Uhlenbeck, Harmonic maps into Lie groups (Classical solutions of the chiral model), J. Differential Geometry, to appear.

[32] J. Wolfson, On minimal surfaces in a Kähler surface of constant holomorphic sectional curvature, Trans. Amer. Math. Soc. 290 (1985) 627-646.

[33] Harmonic maps of $S^{2}$ into $G(2, n: \mathrm{C})$, Complex Differential Geometry and NonLinear Differential Equations, Bowdoin, 1984.

[34] J. C. Wood, Holomorphic differentials and classification theorems for harmonic maps and minimal immersions, Global Riemannian Geometry, Ellis Horwood, Chichester, 1984, $168-176$

[35] H. H. Wu, The equidistribution theory of holomorphic curves, Annals of Math. Studies 64, Princeton University Press, Princeton, NJ, 1970.

UNIVERSITY OF BATH, ENGLAND

UNIVERSITY OF LEEDS, ENGLAND 
